\title{
Ehrlichia SLiM ligand mimetic activates Notch signaling in human monocytes
}

LaNisha L. Patterson ${ }^{1}$, Thangam Sudha Velayutham ${ }^{1}$, Caitlan D. Byerly ${ }^{1}$, Duc Cuong Buil $^{1}$, Jignesh Patel ${ }^{1}$ Veljko Veljkovic ${ }^{6}$, Slobodan Paessler ${ }^{1}$, and Jere W. McBride ${ }^{1-5}$

${ }^{1}$ Departments of Pathology and ${ }^{2}$ Microbiology and Immunology,

${ }^{3}$ Center for Biodefense and Emerging Infectious Diseases,

${ }^{4}$ Sealy Institute for Vaccine Sciences,

${ }^{5}$ Institute for Human Infections and Immunity,

University of Texas Medical Branch, Galveston, TX 77555

${ }^{6}$ Biomed Protection, LLC, Galveston, TX

Correspondence:

Jere W. McBride, Ph.D.

Department of Pathology

University of Texas Medical Branch

Galveston, TX 77555-0609

Tel: (409) 747-2498

Email: jemcbrid@utmb.edu 
1

2

3

4

5

6

7

8

9

\section{Abstract}

Ehrlichia chaffeensis evades innate host defenses by reprogramming the mononuclear phagocyte through mechanisms that involve exploitation of multiple evolutionarily conserved cellular signaling pathways including Notch. This immune evasion strategy is directed in part by tandem repeat protein (TRP) effectors. Specifically, the TRP120 effector activates and regulates Notch signaling through interactions with the Notch receptor and the negative regulator, F-Box and WD repeat domain-containing 7 (FBW7). However, the specific molecular interactions and motifs required for $E$. chaffeensis TRP120-Notch receptor interaction and activation have not been defined. To investigate the molecular basis of TRP120 Notch activation, we compared TRP120 with endogenous canonical/non-canonical Notch ligands and identified a short region of sequence homology within the tandem repeat (TR) domain. TRP120 was predicted to share biological function with Notch ligands, and a function-associated sequence in the TR domain was identified. To investigate TRP120-Notch receptor interactions, colocalization between TRP120 and endogenous Notch-1 was observed. Moreover, direct interactions between full length TRP120, the TRP120 TR domain containing the putative Notch ligand sequence, and the Notch receptor LBR were demonstrated. To molecularly define the TRP120 Notch activation motif, peptide mapping was used to identify an 11-amino acid short linear motif (SLiM) located within the TRP120 TR that activated Notch signaling and downstream gene expression. Peptide mutants of the Notch SLiM or anti-Notch SLiM antibody reduced or eliminated Notch activation and NICD nuclear translocation. This investigation reveals a novel molecularly defined pathogen encoded Notch SLiM mimetic that activates Notch signaling consistent with endogenous ligands. 
bioRxiv preprint doi: https://doi.org/10.1101/2022.01.13.476283; this version posted January 15, 2022. The copyright holder for this preprint (which was not certified by peer review) is the author/funder. All rights reserved. No reuse allowed without permission.

25 Keywords: Ehrlichia; tandem repeat protein; effector; Notch signaling; ligand; short linear motif; 26 molecular mimicry 


\section{Importance}

28 E. chaffeensis infects and replicates in mononuclear phagocytes, but how it evades innate

29 immune defenses of this indispensable primary innate immune cell is not well understood. This

30 investigation reveals the molecular details of a ligand mimicry cellular reprogramming strategy

31 that involves a short linear motif (SLiM) which enables $E$. chaffeensis to exploit host cell

32 signaling to establish and maintain infection. E. chaffeensis TRP120 is a moonlighting effector

33 that has been associated with cellular activation and other functions including ubiquitin ligase

34 activity. Herein, we identify and demonstrate that a SLiM present within each tandem repeat of

35 TRP120 activates Notch signaling. Notch is an evolutionarily conserved signaling pathway

36 responsible for many cell functions including cell fate, development, and innate immunity. The

37 proposed study is significant because it reveals the first molecularly defined pathogen encoded

38 SLiM that appears to have evolved de novo to mimic endogenous Notch ligands. Understanding

39 Notch activation during E. chaffeensis infection provides a model in which to study pathogen

40 exploitation of signaling pathways and will be useful in developing molecularly-targeted

41 countermeasures for inhibiting infection by a multitude of disease-causing pathogens that

42 exploit cell signaling through molecular mimicry. 


\section{Author Summary}

45 E. chaffeensis is a small, obligately intracellular, Gram-negative bacterium that has evolved

46 cellular reprogramming strategies to subvert innate defenses of the mononuclear phagocyte.

47 Ehrlichial TRP effectors interface with the host cell and are involved in pathogen-host interplay

48 that facilitates exploitation and manipulation of cellular signaling pathways; however, the

49 molecular interactions and functional outcomes are not well understood. This study provides

50 molecular insight into a eukaryotic mimicry strategy whereby secreted effectors of obligately

51 intracellular pathogens activate the evolutionarily conserved Notch signaling pathway through a

52 short linear motif ligand mimetic to promote intracellular infection and survival. 


\section{Introduction}

Ehrlichia chaffeensis is a small, obligately intracellular, Gram-negative tick transmitted bacterium (1) that exhibits tropism for mononuclear phagocytes. E. chaffeensis establishes

56 infection through a multitude of cellular reprogramming strategies that involve effector-host

57 interactions resulting the in activation and manipulation of cell signaling pathways to suppress and evade innate immune mechanisms (2-8). The mechanisms whereby $E$. chaffeensis evades host defenses of the macrophage involves exploitation of Wnt and Notch signaling by the tandem repeat protein (TRP) effector, TRP120 (2-6). roles as a nucleomodulin $(9,10)$, a HECT E3 ubiquitin ligase $(2,7,11)$, and as a ligand mimic $(3,5,6,12)$. Previously, we found that TRP120 is involved in a diverse array of host cell interactions including components of signaling and transcriptional regulation associated with Wnt and Notch signaling pathways (8). We have recently shown that TRP120 ubiquitinates the Notch negative regulator FBW7 resulting in increased NICD levels, as well as other FBW7 regulated oncoproteins during infection (2). In addition, we have also demonstrated that $E$. chaffeensis Notch activation results in downregulation of toll-like receptor 2 and 4 expression, likely as an immune evasion mechanism (6). Although we have demonstrated TRP120 activates Notch signaling, the molecular details involved in activation have yet to be defined.

The Notch signaling pathway is evolutionarily conserved and is known to play a critical role in cell proliferation, differentiation, and apoptosis in all metazoan organisms (13-16). Notch activation plays significant roles in various other cellular outcomes, including MHC Class II expansion (17), B- and T- cell development (18), and innate immune mechanisms such as autophagy (19) and apoptosis $(20,21)$. Canonical Notch activation is driven by direct cellmembrane bound receptor-ligand interactions with four Notch receptors (Notch1-4) and 77 canonical Notch ligands, Delta-like (DLL 1,3,4) and Jagged (Jagged/Serrate-1 and 2). Notch 
receptor-ligand interactions occur at the Notch extracellular domain (NECD), specifically at epidermal growth factor-like repeats (EGFs) 11-13, the known ligand binding domain (LBD). Module at the N-terminus of Notch ligands (MNNL) and Delta/Serrate/LAG-2 (DSL) domains in canonical Notch ligands interact with the Notch LBD. Although there is evidence demonstrating the requirement of both N-terminal MNNL and DSL Notch ligand domains for Notch receptor binding, there is little information known about ligand regions/motifs that are necessary for Notch activation $(22,23)$. During canonical Notch activation, ligands expressed on neighboring cells bind the Notch receptor and create a mechanical force at the negative regulatory region (NRR) which triggers several sequential proteolytic cleavages, releasing the Notch intracellular 87 domain (NICD). NICD subsequently translocates to the nucleus and binds to other transcriptional coactivators, including RBPjK and MAML, to activate Notch gene transcription. Notably, secreted non-canonical Notch ligands have also been shown to activate Notch signaling; however, the molecular details of non-canonical Notch ligand-receptor interactions are not well defined.

There are three major classes of protein interaction modules which include globular domains, IDDs, and short linear motifs (SLiMs), all of which have distinct biophysical attributes (24-26). IDDs are 20-50 amino acids in length, are known to be disordered in nature, are located within globular domains or intrinsically disordered protein regions and have transient interactions in the nanomolar range. In comparison, SLiMs are 3-12 amino acids in length, are

97 known to be disordered in nature, located within globular domains or IDDs, and have low 98 micromolar affinity ranges with transient interactions. SLiMs have been shown to evolve de 99 novo for promiscuous binding to various partners $(26,27)$. Ehrlichial TRPs interact with a 100 diverse array of host proteins through several well-known protein-protein interaction 101 mechanisms including post-translational modifications (PTMs), and various protein interaction 102 modules located in intrinsically disordered domains (IDDs) $(7,9,26,28)$. 
Microorganisms have developed mechanisms to survive in the host cell which involve hijacking host cell processes. Molecular mimicry has been well-established as an evolutionary survival strategy utilized by pathogens to disrupt or co-opt host function for infection and survival [26-29]. Studies have determined this occurs through pathogen effectors that mimic eukaryotic

107 host proteins, allowing for pathogens to hijack and manipulate host cellular pathways and 108 functions. SLiMs have been identified as interaction modules whereby eukaryotes and 109 pathogens direct cellular processes through protein-protein interactions $(29,30)$. Recently, we have demonstrated TRP120 is a Wnt ligand mimetic that interacts with host Wnt receptors to activate Wnt signaling (3).

116 investigate molecular host-pathogen interactions involved in repurposing host signaling 117 pathways for infection.

\section{Results}

\section{E. chaffeensis TRP120 shares sequence homology and predicted Notch ligand function.}

120 We have previously shown TRP120 interacts with Notch activating metalloprotease, ADAM17

121 and Notch antagonist FBW7 using yeast-two hybrid analysis (Y2H) (8). We have also shown

122 that TRP120 binds to the promoter region of notch1 using chromatin immunoprecipitation 123 sequencing (ChIP-Seq), and that activation of Notch occurs during infection (6, 10). Notch 124 activation occurs through direct interaction of Notch ligands with the Notch-1 receptor initiating 125 two receptor proteolytic cleavages, resulting in NICD nuclear translocation and subsequent 126 activation of Notch downstream targets. Since TRP120 has been shown to activate the Notch 
signaling pathway, we examined TRP120 sequence homology and correlates of biological functionality with Notch ligands.

NCBI Protein Basic Local Alignment Search Tool (BLAST) was used to identify local similarity between TRP120 and canonical/non-canonical Notch ligand sequences. Sequence homology with a TRP120 tandem repeat (TR) IDD motif, TESHQKEDEIVSQPSSE (aa. 284301), was shown to share sequence homology with several canonical Notch ligands, including Jagged-1, DLL1, DLL4, and non-canonical Notch ligand TSP2 (Fig. 1A). We then used informational spectrum method (ISM) to predict similar functional properties between TRP120 and Notch ligands. ISM is a prediction method that uses the electron ion interaction potential of each amino acid within the primary sequence of proteins to translate the primary sequences into numerical sequences. Translated sequences are then converted into a spectrum using Fourier transform. Cross spectral analysis of the translated sequences is then performed to obtain characteristic frequency peaks that demonstrate if proteins share a similar biological function. TRP120 was predicted to share a similar biological function with canonical Notch ligands, DLL1, 3 and 4, and non-canonical Notch ligand F3 contactin-1, a known adhesion molecule (Figs. S1

142 A-D). To identify the sequence responsible for the identified frequency peaks, reverse Fourier 143 transform of ISM was performed (Fig. 1B). A 35-mer TRP120-TR IDD motif, 144 IVSQPSSEPFVAESEVSKVEQEETNPEVLIKDLQD (aa. 214-248 and 294-328), was associated 145 with characteristic frequency peaks (Figs. 1B and 1C). Collectively, these results indicate that 146 the TRP120 sequence and fundamental biophysical properties of the amino acids are consistent 147 with Notch ligands.

149 Canonical activation of the Notch pathway is known to occur through canonical Notch ligands 150 binding to Notch receptor LBD (EGFs 11-13 in the extracellular domain). To investigate if 151 TRP120 interacts with the Notch-1 receptor LBR (EGFs 1-15), we ectopically expressed GFP- 
152 tagged full length TRP120 (TRP120-FL-GFP) in HeLa cells and probed for endogenous Notch-1

153 to determine colocalization. Pearson's correlation coefficient (PC) and Mander's coefficient (MC)

154 (correlation range +1 to -1 ; 0 represents absence of correlation), was used to quantify the

155 degree of colocalization between TRP120-FL-GFP and Notch-1. Ectopically expressed

156 TRP120-FL-GFP was found to strongly colocalize $(P C=0.897$ and $M C=0.953)$ with

157 endogenous Notch-1 (Fig. 2A). Colocalization of TRP120 and Notch-1 demonstrates that these

158 two proteins are in the same spatial location; however, it does not demonstrate direct protein-

159 protein interaction. To confirm a direct interaction, we utilized pull-down assays of TRP120-FL

160 and Notch-1 LBR. A His-tagged rTRP120-FL (rTRP120-FL-His) construct was incubated with a

161 Fc-tagged recombinant Notch-1 LBR, and a direct protein-protein interaction was demonstrated

162 (Figs. 2B and S2A-B). Thioredoxin (TRX), used as a fusion tag in the pBAD expression vector

containing TRP120 constructs and as a recombinant control, did not interact (Fig. 2B). Based on

sequence homology and ISM data, a short region of sequence homology within the tandem repeat (TR) domain was identified that could be involved in the TRP120 and Notch-1 LBR. To determine if the TRP120-TR was responsible for the previous TRP120 and Notch-1 LBR

167 interaction, we performed a pull-down assay with TRP120-TR and Notch-1 LBR. rTRP120-TR was pulled down with anti-Notch-1 LBR antibody demonstrating a direct interaction with the TR domain (Fig. 2C).

To further confirm direct interaction of TRP120-FL or TRP120-TR and Notch-1 LBR,

171 surface plasmon resonance was performed. An interaction between both rTRP120-FL (Fig. 2D)

172 and rTRP120-TR (Fig. 2E) with Notch-1 LBR was detected in a concentration dependent

173 manner. Fitting the concentration response plots for TRP120-FL and TRP120-TR yielded a $K_{D}$

174 (equilibrium dissociation constant) of $100 \pm 3.5 \mathrm{nM}$ and $120 \pm 2.0 \mathrm{nM}$, respectively (Figs. 2D-E).

175 No interaction was detected between TRX and Notch-1 LBR (Fig. 2F). Additionally, treatment of

176 THP-1 cells with TRP120-coated sulfate, yellow-green microspheres demonstrated 
177

178

179

180

colocalization of TRP120 and Notch-1 (Figs. 2G-H). In comparison, TRX-coated fluorescent microsphere did not colocalize with the Notch-1 receptor (Figs. 2G-H). Together, these binding data reveal TRP120-TR binds the Notch-1 LBR.

\section{E. chaffeensis TRP120-TR domain is required for Notch activation. Both the N-terminal} MNNL and cysteine-rich DSL domain of Notch ligands are known to be required for receptor binding; however, there is little known regarding ligand motifs required for Notch activation. We have previously demonstrated Notch activation occurs in THP-1 cells after stimulation with TRP120-coated beads for 15 min (6). Gene expression levels of notch1, hes1 and hes5 were upregulated after incubation with TRP120-coated beads. To further delineate the TRP120 domain required for Notch activation THP-1 cells or primary human monocytes were treated with soluble purified full length or truncated constructs of recombinant TRP120 (rTRP120-TR and -C-terminus) (Figs. S2A-B). Full length rTRP120 and rTRP120-TR caused NICD nuclear translocation 2 h post-treatment (Figs. 3A-B). NICD nuclear translocation was not observed in untreated cells, cells treated with TRX or rTRP120-C-terminal soluble proteins (Figs. 3A-B). Collectively, these data demonstrate the requirement of TRP120-TR for Notch activation.

\section{E. chaffeensis TRP120-TR Notch ligand IDD-mimetic activates Notch.}

To determine if Notch is activated by a TRP120-TR Notch mimetic IDD motif, several TRP120TR synthetic peptides were generated (Fig. 4A). THP-1 cells or primary human monocytes were treated with TRP120-TR IDD peptides or scrambled negative control peptide for $2 \mathrm{~h}$. A 35-aa TRP120-TR IDD motif (TRP120-N1-P3) caused nuclear translocation (Figs. 4B and C). Importantly, the identified IDD contained a motif identified in both sequence homology and ISM data (Fig. 1C). Inhibition of Notch signaling by DAPT, a y-secretase inhibitor, abrogated Notch activation with TRP120-N1-P3 treatment, indicating that TRP120-N1-P3 directly binds to the Notch-1 receptor for Notch activation (Fig. 4B). To confirm Notch activation by TRP120-N1-P3, 
203

204

205

206

207

208

209

210

211

212

213

214

215

216

217

218

219

220

221

222

223

224

225

226

227

gene expression levels of Notch downstream targets were examined by human Notch signaling pathway array analysis. In comparison to untreated THP-1 cells, a significant increase in Notch downstream targets, including HES1, HES5, HEY1 and HEY2 gene expression levels occurred in TRP120-N1-P3 treated cells (Figs. 5A-B, Table S1A). Interestingly, Notch gene expression by TRP120-N1-P3 treatment was increased in a concentration-dependent manner (Fig. 5B, Table S1A). Importantly, rJagged-1 also demonstrated similar upregulation of Notch genes in a concentration-dependent manner (Fig. S3). These data demonstrate that a TRP120 IDD mimetic motif is responsible for TRP120 Notch activation.

\section{E. chaffeensis TRP120-TR Notch ligand SLiM mimetic activates Notch.}

It is well-documented that SLiMs are found in two general groups; posttranslational modification (PTM) motifs or ligand motifs that mediate binding events. We have previously identified a functional TRP120 HECT E3 ligase catalytic motif located in the C-terminus $(2,7)$ and have recently identified a TRP120-TR Wnt SLiM mimetic motif (3). To determine if the TRP120-TR Notch mimetic motif could be a SLiM (3-12 aa), overlapping TRP120-TR synthetic peptides that span the identified 35-aa TRP120-TR IDD motif were synthesized (Fig. 6A). Treatment with P4 and P5 TRP120-TR Notch mimetic SLiM peptides in THP-1 cells did not result in NICD nuclear translocation (Fig. 6B); however, TRP120-TR Notch mimetic SLiM P6 (TRP120-N1-P6) located at the C-terminus resulted in NICD nuclear translocation (Fig. 6B). TRP120-N1-P6 was also shown to cause NICD nuclear translocation in primary human monocytes (Fig. 6C).

Furthermore, pre-treatment of DAPT inhibited TRP120-N1-P6 Notch activation (Fig. 6B). Upregulation of Notch downstream targets occurred with TRP120-N1-P6 treatment in a concentration dependent manner (Figs. 7A-B, Table S1B), as previously shown with the TRP120-N1-P3 peptide. In comparison, TRP120-N1-P5 peptide treatment, did not result in significant upregulation of Notch gene expression (Fig. 7B). 
To confirm that TRP120-N1-P6 is required for Notch activation, a TRP120-N1-P3 mutant peptide (dmut) (Fig. 6A) without the TRP120-N1-P6 motif was tested. THP-1 cells stimulated with TRP120-N1-dmut exhibited abrogated Notch activation as demonstrated by NICD translocation (Fig. 6B). To determine the minimal residues required in the TRP120-TR Notch

232 mimetic SLiM, alanine mutagenesis was used to determine the contribution of specific residues

233 to Notch activation (Fig. 8A, blue boxes). Mutated residues were selected based on sequence

234 homology and ISM data. Mutants (dmut-1 -2, -3 and -4) exhibited reduced Notch activation as

235 determined by NICD translocation, but only the TRP120-N1-dmut peptide resulted in full

236 abrogation of NICD nuclear translocation (Fig. 8A). Collectively, these data demonstrate that the 237 TRP120-N1-P6 SLiM is a Notch mimetic.

TRP120 Notch SLiM antibody blocks E. chaffeensis Notch activation. To investigate whether the TRP120 Notch mimetic is solely responsible for Notch activation by E. chaffeensis,

241 THP-1 cells were pre-treated with a purified rabbit polyclonal antibody generated against the

242 TRP120-N1-P6 SLiM and subsequently infected with E. chaffeensis for 2 h. Negative pre-

243 immune serum was used as a negative control. NICD nuclear translocation was determined in $244 \alpha-T R P 120-N 1-P 6$ SLiM or negative pre-immune serum treated cells. THP-1 cells treated with $\alpha-$ 245 TRP120-N1-P6 SLiM did not display NICD nuclear translocation, in comparison to the negative 246 pre-immune serum control (Fig. 8B). These data suggests that TRP120-N1-P6 SLiM is the only 247 Notch mimic involved in Notch activation by E. chaffeensis.

\section{Discussion}

We have previously demonstrated TRP120-host interactions to occur with a diverse 251 array of host cell proteins associated with conserved signaling pathways, including Wnt and 252 Notch (8). Two proteins shown to interact with TRP120 were the Notch metalloprotease, a 253 disintegrin and metalloprotease domain (ADAM17), and a Notch antagonist, F-box and WD 
254 repeat domain-containing 7 (FBW7). In addition, we have demonstrated that secretion of $E$.

255 chaffeensis TRP120 activates Notch signaling to downregulate TLR2/4 expression for

256 intracellular survival. Moreover, Keewan et al, demonstrated upregulation of Notch-1, IL-6 and

257 MCL-1 during M. avium paratuberculosis infection (37). Notch-1 signaling was shown to

258 modulate macrophage polarization and immune defense against during infection, but the

259 molecular mechanisms were not defined; however, the molecular mechanisms utilized for

260 TRP120 Notch activation have not been previously studied (6). In this study, we investigated the

261 molecular interactions involved in TRP120 Notch activation and have defined a TRP120 Notch

262 SLiM mimetic responsible for Notch activation.

Molecular mimicry has been well-established as an evolutionary survival strategy utilized disordered class of protein interaction motifs that have been shown to evolve de novo for promiscuous binding to various partners and have been documented as a host hijacking mechanism for pathogens $(26,29,30)$. Although SLiM mimicry has been established as a mechanism utilized by pathogens to repurpose host cell functions for survival, a Notch ligand mimic has never been defined. been previously described as important for TRP120's moonlighting capabilities $(9,12)$. Within

273 these intrinsically disordered domains are various SLiMs responsible for TRP120 multi-

274 functionality. We have recently defined a novel TRP120 repetitive SLiM that activates Wnt 275 signaling to promote E. chaffeensis infection (3). In the current study, we have also determined

276 TRP120-TR as the domain also responsible for Notch activation. Sequence homology studies 277 and Information Spectrum Method (ISM) have shown sequence similarity and similar biological 278 function between TRP120 and endogenous Notch ligands. ISM is a virtual spectroscopy method 
utilized to predict if proteins share a similar biological function based on the electron-ion interaction potential of amino acids, and only requires the nucleotide sequence of each protein.

281 It was recently used to determine prediction of potential receptor, natural reservoir, tropism and 282 therapeutic/vaccine target of SARS-CoV-2 (38). Our results demonstrate a shared sequence 283 similarity and biological function with both canonical and non-canonical Notch ligands that 284 occurs within the tandem repeat domain of TRP120 (TRP120-TR). Both sequence homology 285 and ISM studies identified specific tandem repeat sequences that are functionally associated 286 with endogenous Notch ligands and range between 20-35 amino acids in size. This data suggested that intrinsically disordered regions found within the TRP120-TR domain are responsible for Notch ligand mimic function and direct effector-host protein interaction with the Notch receptor. receptor $(39,40)$. Canonical Notch ligands are known to contain a DSL domain that is important 292 for Notch binding and activation, but a conserved activation motif has not been defined.

293 Colocalization of TRP120 with Notch-1 was previously shown to occur during E. chaffeensis 294 infection (6); however, a direct interaction was not previously shown using yeast-two hybrid (8), 295 possibly due to limitations of this technique with protein interactions involving membrane 296 proteins $(6,41)$. Using pull down, SPR and protein-coated fluorescent microsphere approaches, 297 we further studied TRP120-Notch-1 interaction and found direct binding occurs through 298 TRP120-TR at a Notch-1 LBR (EGFs 1-15). TRP120-TR and Notch-1 LBR interaction occurred 299 at an affinity of $120 \pm 2.0 \mathrm{nM}$, indicating a strong protein-protein interaction. Numerous structural 300 studies of interactions of Notch with endogenous ligands have shown low affinity interactions 301 between Notch Jag or DLL ECDs (42-44). One study demonstrated weak affinities between 302 Notch-1 with an engineered high affinity Jag-1 variant $\left(K_{D}=5.4 \mu M\right)$ and DLL4 (12.8 $\left.\mu M\right)(39)$. 303 The higher binding affinity of TRP120-TR in comparison to canonical Notch ligands suggests 
that the four tandemly repeated motifs folds in a structure that potentiates binding between TRP120 and Notch-1. In addition, stimulating THP-1 cells and primary monocytes with TRP120TR resulted in NICD nuclear translocation, indicating that TRP120-TR is the TRP120 domain responsible for Notch activation. Interestingly, TRP120-Fzd5 interaction also occurred through the tandem repeat domain and supports our current findings that TRP120-host protein interactions occur within regions of the tandem repeat domain, likely due to its disordered nature (3).

Secreted and membrane-bound proteins have been shown to activate Notch signaling.

312 These non-canonical Notch ligands lack the DSL domain but still have the ability to modify

313 Notch signaling. Some of the non-canonical Notch proteins contain EGF-like domains; however,

314 others share very little sequence similarity to endogenous Notch ligands $(23,45)$. TSP2 is a

315 secreted mammalian protein containing EGF-like domains. TSP2 was found to potentiate Notch

316 signaling by direct Notch-3/Jagged1 binding (46). Furthermore, TSP2 binds directly to purified

317 Notch-3 protein containing EGF-like domains 1-11, suggesting a direct interaction. Non-

318 canonical Notch ligand TSP2 was found to share significant sequence homology within the

319 TRP120-TR sequence. Homologous regions included the identified TRP120-TR Notch SLiM

320 mimetic. Although TSP2 has been identified as a secreted, non-canonical Notch ligand, there

321 has been no activating motif identified to date. F3/contactin1, another identified secreted non-

322 canonical Notch ligand, does not contain DSL or EGF-like domains; however, it activates the

323 Notch signaling pathway through the Notch-1 receptor (47). TRP120 was found to share

324 biological function with F3/contactin1 by ISM. F3/contactin1 has been demonstrated to bind to

325 Notch-1 at two different locations within the NECD and activates Notch signaling when

326 presented as purified soluble protein (47). Therefore, Notch activation by secreted, non-

327 canonical Notch ligands has been demonstrated; however, more insight into the molecular 
details of those interactions needs to be elucidated. This study provides new insight regarding non-canonical Notch ligand activation of the Notch signaling pathway.

SLiMs have been identified in secreted effector proteins of intracellular bacterial pathogens, including Ehrlichia, Anaplasma phagocytophilum (48), Legionella pneumophila (4951) and Mycobacterium tuberculosis (52). This investigation identifies a novel Notch SLiM (11 aa) that can activate Notch signaling as a soluble ligand. Complete NICD nuclear translocation was previously shown to occur at $2 \mathrm{~h}$ post-infection (6), indicating that NICD nuclear translocation during E. chaffeensis infection is a result of TRP120-TR Notch ligand SLiM mimetic interaction with the Notch-1 receptor. In addition, Notch signaling pathway genes were upregulated at $24 \mathrm{~h}$ in TRP120-TR Notch mimetic SLiM-treated THP-1 cells. These data are consistent with our previous findings where we detected upregulation of Notch signaling pathway components and target genes during $E$. chaffeensis infection at $12,24,48$, and 72 h.p.i., with maximum changes in Notch gene expression occurring at 24 h.p.i (6). Furthermore, during E. chaffeensis infection, TRP120 mediated ubiquitination and proteasomal degradation of Notch negative regulator, FBW7 begins at 24 h.p.i. and gradually decreases during late stages of infection (2). Both TRP120 and FBW7 are localized to the nucleus beginning at 24 h.p.i., suggesting that TRP120-degradadtion of FBW7 assists in upregulation of Notch downstream targets at this timepoint (2).

Interestingly, both the TRP120 Notch memetic IDD (TRP120-N1-P3) and SLiM (TRP120-N1-P6) resulted in concentration-dependent upregulation of Notch downstream targets. Similar to our findings, studies have shown that the Notch pathway can induce heterogenous phenotypic responses in a Notch ligand or NICD dose dependent manner. Klein et al. demonstrated that high levels of Notch ligands can induce a ligand inhibitory effect, while lower levels of Notch ligand activate Notch signaling activity (53). Similarly, Semenova D et al. has shown that NICD and Jag1 transduction increases osteogenic differentiation in a dose- 
dependent manner; however high dosage of NICD and Jag1 decreases osteogenic differentiation efficiency (54). Furthermore, Gomez-Lamarca et al. has shown that NICD dosage can influence CSL-DNA binding kinetics, NICD dimerization, and chromatin opening to strengthen transcriptional activation (55). Therefore, an increase in Notch ligand-receptor interaction may lead to increased NICD release and Notch signaling strength. mimetic is required for Notch activation. Importantly, SLiMs are known to have low-affinity, transient protein-protein interactions within the low-micromolar range (26). In this case, the repeated TRP120-TR Notch ligand SLiM mimetic motif may cause TRP120 to fold in a tertiary structure upon binding to the Notch-1 receptor that stabilizes the TRP120-Notch-1 interaction. Based on this data, E. chaffeensis TRP120 could be used as a model to study SLiMs within intrinsically disordered effector proteins that are utilized for host exploitation by other intracellular bacterial pathogens.

To demonstrate that TRP120-TR Notch ligand SLiM mimetic motif is solely responsible for $E$. chaffeensis activation of Notch, we generated an antibody against the mimetic epitope to block E. chaffeensis TRP120-Notch-1 binding. Our results demonstrated antibody blockade of Notch activation by E. chaffeensis, rTRP120 and the TRP120-TR Notch ligand SLiM peptide.

370 This data strongly supports the conclusion that the TRP120-TR Notch ligand SLiM mimetic is 371 responsible for $E$. chaffeensis Notch activation and may provide a new E. chaffeensis

372 therapeutic target. Hence, this study serves to provide insight into the molecular details of how

373 Notch signaling is modulated during E. chaffeensis infection and may serve as a model for other 374 pathogens.

Further outstanding questions regarding regulation of the Notch signaling pathway

376 during E. chaffeensis remain. We have recently demonstrated maintenance of Notch activation 377 is linked to TRP120-mediated ubiquitination and proteasomal degradation of tumor suppressor 
FBW7, a Notch negative regulator (2). However, other potential Notch regulators may serve as a target for TRP120-medidated ubiquitination for constitutive Notch activation during infection. Suppressor of Deltex $[\mathrm{Su}(\mathrm{dx})]$ is an E3 ubiquitin ligase that serves as another negative regulator of Notch signaling by degrading Deltex, a positive regulator of Notch signaling (56). Su(dx) may serve as another target of TRP120-mediated ubiquitination to maintain Notch activity during $E$. chaffeensis infection. Furthermore, how secreted non-canonical Notch ligands are able to cause separation between the NICD and NECD remains unknown. TRP120 causes Notch activation, resulting in upregulation of Notch downstream targets; however, the mechanism of how the S2 exposure for ADAM cleavage is not understood. Future crystallography studies on TRP120 and Notch-1 interaction may provide more insight into these structural details required for TRP120N1-P6 SLiM Notch activation $(57,58)$.

In conclusion, we have demonstrated $E$. chaffeensis Notch activation is initiated by a TRP120 Notch SLiM mimetic. Our findings have identified a pathogen protein host mimic to repurpose the evolutionarily conserved Notch signaling pathway for intracellular survival. This study gives more insight into how obligate intracellular pathogens, with small genomes have evolved host mimicry modules de novo to exploit conserved signaling pathways to suppress

394 innate defenses to promote infection.

\section{Materials and Methods}

397 Cell culture and cultivation of $E$. chaffeensis. Human monocytic leukemia cells (THP-1; 398 ATCC TIB-202) were propagated in RPMI media (ATCC) containing $2 \mathrm{mM}$ L-glutamine, $10 \mathrm{mM}$ 399 HEPES, $1 \mathrm{mM}$ sodium pyruvate, $4500 \mathrm{mg} / \mathrm{L}$ glucose,1500 mg/L sodium bicarbonate, 400 supplemented with $10 \%$ fetal bovine serum (FBS; Invitrogen) at $37^{\circ} \mathrm{C}$ in $5 \% \mathrm{CO}_{2}$ atmosphere. E. 401 chaffeensis (Arkansas strain) was cultivated in THP-1 cells. Host cell-free E. chaffeensis was prepared by rupturing infected THP-1 cells or primary human monocytes with sterile glass 
403

404

405

406

407

408

409

410

411

412

413

414

415

416

417

418

419

420

421

422

423

424

425

426

427

beads $(1 \mathrm{~mm})$ by vortexing. Infected THP-1 cells were harvested and pelleted by centrifugation at $500 \times g$ for $5 \mathrm{~min}$. The pellet was resuspended in sterile phosphate-buffered saline (PBS) in a 50-ml tube containing glass beads and vortexed at moderate speed for $1 \mathrm{~min}$. The cell debris was pelleted at $1,500 \times g$ for $10 \mathrm{~min}$, and the supernatant was further pelleted by high-speed centrifugation at $12,000 \times g$ for $10 \mathrm{~min}, 4^{\circ} \mathrm{C}$. The purified ehrlichiae were resuspended in fresh RPMI media and utilized as needed.

Human PBMC and primary monocyte isolation. Primary human monocytes were isolated from $125 \mathrm{ml}$ of human blood obtained from Gulf Coast Regional Blood Center (Houston, TX). Blood was diluted in RMPI media and separated by density gradient separation on Ficoll at 2000 rpm for 20 minutes. The plasma was removed from the separated sample and the buffy coat was collected. Buffy coat was diluted with DPBS containing $2 \%$ FBS and $1 \mathrm{mM}$ EDTA and centrifuged at $1500 \mathrm{rpm}$ for 15 minutes. Supernatant was removed and all cells were combined and mixed carefully. Combined cells were then centrifuged at 1500 rpm for 10 minutes and supernatant was removed. Cells were resuspended into $1 \mathrm{~mL}$ of DPBS containing $2 \%$ FBS and $1 \mathrm{mM}$ EDTA. Cells were then diluted to $5 \times 10^{7} / \mathrm{mL}$ concentration, and monocytes were separated by the EasySep Human Monocyte Enrichment Kit w/o CD16 depletion (Stemcell \#19058) according to the manufacturers protocol. Primary human monocytes were then cultured in RPMI media (ATCC) containing 2 mM L-glutamine, 10 mM HEPES, 1 mM sodium pyruvate, $4500 \mathrm{mg} / \mathrm{L}$ glucose, $1500 \mathrm{mg} / \mathrm{L}$ sodium bicarbonate, supplemented with $10 \%$ fetal bovine serum (FBS; Invitrogen) at $37^{\circ} \mathrm{C}$ in $5 \% \mathrm{CO}_{2}$ atmosphere.

Antibodies and Reagents. Primary antibodies used in this study for immunofluorescence microscopy, Western blot analysis, and pull-down assays include monoclonal rabbit $\alpha$-Notch1 (3608S; Cell Signaling Technology, Danvers MA), polyclonal rabbit a-Notch1, intracellular (071231; Millipose Sigma, Billerica, MA), rabbit a-TRP120-I1 (59) polyclonal rabbit $\alpha$-TRX (T0803; Sigma-Aldrich, Saint Louis, MO). Polyclonal rabbit anti-TRP120 antiserum was commercially 
generated against a TRP120 epitope inclusive of aa. 290-301 (GenScript, Piscataway, NJ). Synthetic peptides used in this study were commercially generated (Genscript, Piscataway, NJ).

Sequence Homology. Genome and transcriptome sequences encoding E. chaffeensis TRP120 and Homo sapiens Notch ligand proteins were recovered using BLAST searches with the online version at the NCBI website. Sequences were submitted to NCBI Protein BLAST and ClustalW2 sequence databases for sequence alignment.

Informational Spectrum Method (ISM). ISM analyzes the primary structure of proteins by assigning a physical parameter which is relevant for the protein's biological function $(38,60)$. Each amino acid in TRP120 and Notch ligand sequences was given a value corresponding to its electron-ion interaction potential (EIIP), which determines the long-range properties of biological molecules. The value of the amino acids within the protein were Fourier transformed to provide

439 a Fourier spectrum that is representative of the protein, resulting in a series of frequencies and 440 amplitudes. The frequencies correspond to a physico-chemical property involved in the 441 biological activity of the protein. Comparison of proteins is performed by cross-spectra analysis.

442 Proteins with similar spectra were predicted to have a similar biological function. Inverse Fourier 443 Transform was performed to identify the sequence responsible for obtained signals at a given 444 frequency.

445 Transfection. HeLa cells $\left(1 \times 10^{6}\right)$ were seeded in a $60 \mathrm{~mm}$ culture dish $24 \mathrm{~h}$ prior to 446 transfection. AcGFP-TRP120 or AcGFP-control plasmids were added to OptiMem and 447 Lipofectamine 2000 mixture and incubated for $20 \mathrm{~min}$ at $37^{\circ} \mathrm{C}$. Lipofectamine/plasmid mixtures 448 were added to HeLa cells and incubated for $4 \mathrm{~h}$ at $37^{\circ} \mathrm{C}$. Media was aspirated $4 \mathrm{~h}$ post449 transfection and fresh media was added to each plate and incubated for $24 \mathrm{~h}$.

450 Pull Down Assay. Recombinant His-tagged TRP120 (10 $\mu \mathrm{g})$ and Notch-1 (10 $\mu \mathrm{g})$ (Sino 451 Biological) were incubated with Ni-NTA beads alone, or in combination, for $4 \mathrm{~h}$ at $4^{\circ} \mathrm{C}$. 
452 Supernatants were collected and the Ni-NTA beads were washed 5X with $10 \mathrm{mM}$ imidazole 453 wash buffer. Proteins were eluted off with $200 \mathrm{mM}$ imidazole elution buffer and binding 454 determined by Western blot analysis.

455 Immunofluorescent Confocal Microscopy. THP-1 cells $\left(2 \times 10^{6}\right)$ were treated with full length 456 or truncated constructs (-TR or -C terminus) of recombinant TRP120, or TRP120 peptides for 2 $457 \mathrm{~h}$ at $37^{\circ} \mathrm{C}$. Cells were collected and fixed using $4 \%$ formaldehyde, washed with $1 \mathrm{X}$ PBS and permeabilized and blocked in $0.5 \%$ Triton X-100 and 2\% BSA in PBS for 30 min. Cells were washed with PBS and probed with polyclonal rabbit $\alpha$-Notch-1, intracellular (1:100) (Millipore Sigma, MA) or monoclonal rabbit $\alpha-$ Notch1 (3608S; Cell Signaling Technology, Danvers MA) for $1 \mathrm{~h}$ at room temperature. Cells were washed with PBS and probed with Alexa Fluor 568 rabbit anti-goat $\lg G(\mathrm{H}+\mathrm{L})$ for $30 \mathrm{~min}$ at room temperature, washed and then mounted with ProLong Gold antifade reagent with DAPI (Molecular Probes, OR). Slides were imaged on a Zeiss LSM 880 confocal laser scanning microscopy. Pearson's correlation coefficient and Mander's correlation coefficient was generated by ImageJ software to quantify the degree of colocalization between fluorophores.

Protein-coated fluorescent microsphere assay. TRP120 and TRX recombinant proteins were desalted using Zeba spin desalting columns (Thermo Fisher Scientific, MA) as indicated by the manufacturer protocol. Protein abundance of desalted recombinant protein was assessed by 470 bicinchoninic acid assay (BCA assay). One-micrometer, yellow-green (505/515), sulfate 471 FluoSpheres (Life Technologies, CA) were first equilibrated with 40 $\mu \mathrm{M}$ of MES buffer followed 472 by incubation with $10 \mu \mathrm{g}$ of desalted TRP120 or TRX recombinant protein in $40 \mu \mathrm{M}$ MES (2-(N473 morpholino) ethanesulfonic acid) buffer for $2 \mathrm{~h}$ at room temperature on a rotor. TRP120 or TRX 474 coated FluoSpheres were washed twice with $40 \mu \mathrm{M}$ MES buffer at $12,000 \times \mathrm{g}$ for 5 mins and 475 then resuspended in RPMI media. To determine TRP120 or TRX protein coating of 476 FluoSpheres, dot blotting of FluoSpheres samples was performed after protein coating using $\alpha$ - 
477 TRX or $\alpha$-TRP120 antibodies. $8 \times 10^{5} \mathrm{THP}-1$ cells/well were plated in a 96-well round bottom 478 plate, and the TRP120 or TRX coated FluoSpheres were added to each well at approximately 5 479 beads/cell. The cell and protein-coated FluoSpheres were incubated between $5-60$ mins at $37^{\circ} \mathrm{C}$ 480 with $5 \% \mathrm{CO}^{2}$, collected and unbound beads were washed twice with $1 \mathrm{X}$ PBS, followed by 481 fixation by cytospin for 15 mins. Cell samples were then processed for analysis by 482 immunofluorescent confocal microscopy, as previously mentioned. FluoSpheres are light483 sensitive, therefore all steps were performed in the dark.

484 Quantitative Real-time PCR. The human Notch signaling targets PCR array profiles the 485 expression of 84 Notch pathway-focused genes to analyze Notch pathway status. PCR arrays were performed according to the PCR array handbook from the manufacturer. Briefly, 487 uninfected and E. chaffeensis-infected or Notch mimetic peptide-treated THP-1 cells were collected at 24 and $48 \mathrm{~h}$ intervals and RNA purification with minor modifications, cDNA synthesis and real-time PCR were performed as previously described (3).

490 Western Blot Analysis. Cells were lysed in RIPA lysis buffer (0.5M Tris- $\mathrm{HCl}, \mathrm{pH} 7.4,1.5 \mathrm{M}$ $491 \mathrm{NaCl}, 2.5 \%$ deoxycholic acid, 10\% NP-40, 10mM EDTA) containing protease inhibitor cocktail 492 for $30 \mathrm{~min}$ at $4^{\circ} \mathrm{C}$. Lysates were then cleared by centrifugation and protein abundance assessed 493 by bicinchoninic acid assay (BCA assay). Samples were added to Laemelli buffer then boiled for $4945 \mathrm{~min}$. Lysates were then subjected to SDS-PAGE followed by transfer to nitrocellulose 495 membrane. Membranes were blocked for $1 \mathrm{~h}$ in $5 \%$ nonfat milk diluted in TBST and then 496 exposed to $\alpha$-TRP120, $\alpha-\operatorname{TRX}$ or $\alpha-$ Notch-1 primary antibodies overnight. Membranes were 497 washed three times in Tris-buffered saline containing 1\% Triton (TBST) for 30 min followed by 1 $498 \mathrm{~h}$ incubation with horseradish peroxidase-conjugated anti-rabbit and anti-mouse secondary 499 antibodies (SeraCare, Milford, MA) (diluted 1:10,000 in 5\% nonfat milk in TBST). Proteins were 500 visualized with ECL via Chemi-doc2 and densitometry was measured with VisionWorks Image 501 Acquisition and Analysis Software. 
Surface Plasmon Resonance. SPR was performed using a BIAcore T100 instrument with nitrilotriacetic acid (NTA) sensor chip. Purified polyhistidine-tagged, full-length, rTRP120-TR, rTRX and human rNotch-1 Fc Chimera Protein, CF (R\&D Systems, MN) were dialyzed in running buffer (100 mM sodium phosphate [pH 7.4], $400 \mathrm{mM} \mathrm{NaCl,} 40 \mu \mathrm{M}$ EDTA, 0.005\%

$506[\mathrm{vol} / \mathrm{vol}])$. Briefly, each cycle of running started with charging the NTA chip with $500 \mu \mathrm{M}$ of $\mathrm{NiCl}^{2}$.

507 Subsequently, purified polyhistidine-tagged, full-length, truncated rTRP120 proteins, or rTRX

$508(0.1 \mu \mathrm{M})$ were immobilized on the NTA sensor as ligand on flow cell 2. Immobilization was 509 carried out at $25^{\circ} \mathrm{C}$ at a constant flow rate of $30 \mu \mathrm{l} / \mathrm{min}$ for $100 \mathrm{~s}$. Varying concentrations of 510 Notch1-NECD constructs $(0-800 \mathrm{nM})$ were injected over sensor surfaces as analyte with 511 duplicates along with several blanks of running buffer. Injections of analyte were carried out at a 512 flow rate of $30 \mu \mathrm{l} / \mathrm{min}$ with contact time of $360 \mathrm{~s}$ and a dissociation time of $300 \mathrm{~s}$. Finally, the 513 NTA surface was regenerated by using $350 \mathrm{mM}$ EDTA. Readout included a sensogram plot of 514 response against time, showing the progress of the interaction. Curve fittings were done with 515 the 1:1 Langmuir binding model with all fitting quality critique requirements met. The binding 516 affinity $\left(\mathrm{K}_{\mathrm{D}}\right)$ was determined for all interactions by extracting the association rate constant and 517 dissociation rate constant from the sensorgram curve $\left(K_{D}=K d / K a\right)$ using the BIAevaluation 518 package software.

519 TRP120 Antibody Inhibition of E. chaffeensis Notch activation. Host cell-free E. chaffeensis 520 was pre-treated with $5-10 \mu \mathrm{g} / \mathrm{ml}$ of polyclonal rabbit anti-TRP120 antibody generated against the 521 TRP120 Notch mimetic SLiM (aa. 284-301), or purified IgG antibody. The cell-free $E$. 522 chaffeensis/antibody mixture was then added to THP-1 cells $\left(5 \times 10^{5}\right)$ in a 12-well plate for $2 \mathrm{~h}$. 523 Samples were collected, washed with PBS and prepared for IFA.

524 TRP120 Protein Expression and Purification. Full length or truncated constructs of rTRP120, 525 or rTRX control were expressed in a pBAD expression vector, which has been previously 526 optimized by our laboratory $(59,61,62)$. Recombinant TRP120 full length, truncated constructs, 
527 and rTRX were purified via nickel-nitrilotriacetic acid (Ni-NTA) purification system. All

528 recombinant proteins were dialyzed via PBS and tested for bacterial endotoxins using the

529 Limulus Amebocyte Lysate (LAL) test.

530 Statistical Analysis. All data are represented as the means \pm standard deviation (SD) of data

531 obtained from at least three independent experiments done with triplicate biological replicates,

532 unless otherwise indicated. Analyses were performed using a two-way ANOVA or two-tailed

533 Student's $t$-test (GraphPad Prism 6 software, La Jolla, CA). A P-value of $<0.05$ was considered

534 statistically significant. 


\section{Acknowledgments}

536 We thank the UTMB Solution Biophysics Laboratory and the Optical Microscopy Core for

537 assistance with confocal microscopy. This work was supported by the National Institute of 538 Allergy and Infectious Diseases grants Al158422, Al149136 and Al126144 to J.W.M., a UTMB 539 McLaughlin Endowment Predoctoral Fellowship to L.L.P., an NIH 1F31Al152424 fellowship to 540 L.L.P., and a T32A1007526-20 biodefense training fellowship to C.D.B. 


\section{References}

542 1. Jaworski DC, Cheng C, Nair ADS, Ganta RR. 2017. Amblyomma americanum ticks 543 infected with in vitro cultured wild-type and mutants of Ehrlichia chaffeensis are

2. Wang JY, Zhu B, Patterson LL, Rogan MR, Kibler CE, McBride JW. 2020. Ehrlichia chaffeensis TRP120-mediated ubiquitination and proteasomal degradation of tumor suppressor FBW7 increases oncoprotein stability and promotes infection. PLoS Pathog 16:e1008541.

3. Rogan MR, Patterson LL, Byerly CD, Luo T, Paessler S, Veljkovic V, Quade B, McBride JW. 2021. Ehrlichia chaffeensis TRP120 Is a Wnt Ligand Mimetic That Interacts with Wnt Receptors and Contains a Novel Repetitive Short Linear Motif That Activates Wnt Signaling. mSphere 6.

4. Luo T, Dunphy PS, Lina TT, McBride JW. 2015. Ehrlichia chaffeensis exploits canonical and noncanonical host Wnt signaling pathways to stimulate phagocytosis and promote intracellular survival. Infect Immun 84:686-700.

5. Lina TT, Luo T, Velayutham TS, Das S, McBride JW. 2017. Ehrlichia activation of WntPI3K-mTOR signaling inhibits autolysosome generation and autophagic destruction by the mononuclear phagocyte. Infect Immun 85.

6. Lina TT, Dunphy PS, Luo T, McBride JW. 2016. Ehrlichia chaffeensis TRP120 activates canonical Notch signaling to downregulate TLR2/4 expression and promote intracellular survival. MBio 7.

7. Zhu B, Das S, Mitra S, Farris TR, McBride JW. 2017. Ehrlichia chaffeensis TRP120 moonlights as a HECT E3 ligase involved in self and host ubiquitination to influence protein interactions and stability for intracellular survival. Infect Immun doi:10.1128/IAI.00290-17.

8. Luo T, Kuriakose JA, Zhu B, Wakeel A, McBride JW. 2011. Ehrlichia chaffeensis TRP120 interacts with a diverse array of eukaryotic proteins involved in transcription, signaling, and cytoskeleton organization. Infect Immun 79:4382-91.

9. Klema VJ, Sepuru KM, Fullbrunn N, Farris TR, Dunphy PS, McBride JW, Rajarathnam $\mathrm{K}$, Choi KH. 2018. Ehrlichia chaffeensis TRP120 nucleomodulin binds DNA with disordered tandem repeat domain. PLoS One 13:e0194891.

10. Zhu B, Kuriakose JA, Luo T, Ballesteros E, Gupta S, Fofanov Y, McBride JW. 2011. Ehrlichia chaffeensis TRP120 binds a G+C-rich motif in host cell DNA and exhibits eukaryotic transcriptional activator function. Infect Immun 79:4370-81.

11. Mitra S, Dunphy PS, Das S, Zhu B, Luo T, McBride JW. 2018. Ehrlichia chaffeensis TRP120 effector targets and recruits host polycomb group proteins for degradation to promote intracellular infection. Infect Immun 86.

12. Byerly CD, Patterson LL, McBride JW. 2021. Ehrlichia TRP effectors: moonlighting, mimicry and infection. Pathog Dis 79.

13. Baonza A, Garcia-Bellido A. 2000. Notch signaling directly controls cell proliferation in the Drosophila wing disc. Proc Natl Acad Sci U S A 97:2609-14.

14. Urbanek K, Lesiak M, Krakowian D, Koryciak-Komarska H, Likus W, Czekaj P, Kusz D, Sieron AL. 2017. Notch signaling pathway and gene expression profiles during early in vitro differentiation of liver-derived mesenchymal stromal cells to osteoblasts. Lab Invest 97:1225-1234.

15. Zweidler-McKay PA, He Y, Xu L, Rodriguez CG, Karnell FG, Carpenter AC, Aster JC, Allman D, Pear WS. 2005. Notch signaling is a potent inducer of growth arrest and apoptosis in a wide range of B-cell malignancies. Blood 106:3898-906.

16. Artavanis-Tsakonas S, Rand MD, Lake RJ. 1999. Notch signaling: cell fate control and signal integration in development. Science 284:770-6. 
630

631

632

633

634

635

636

637

638

639

17. Nakano N, Nishiyama C, Yagita H, Koyanagi A, Ogawa H, Okumura K. 2011. Notch1mediated signaling induces $\mathrm{MHC}$ class II expression through activation of class II transactivator promoter III in mast cells. J Biol Chem 286:12042-8.

18. Hoyne GF. 2003. Notch signaling in the immune system. J Leukoc Biol 74:971-81.

19. Sarin A, Marcel N. 2017. The NOTCH1-autophagy interaction: Regulating self-eating for survival. Autophagy 13:446-447.

20. Palaga T, Ratanabunyong S, Pattarakankul T, Sangphech N, Wongchana W, Hadae Y, Kueanjinda P. 2013. Notch signaling regulates expression of $\mathrm{Mcl}-1$ and apoptosis in PPD-treated macrophages. Cell Mol Immunol 10:444-52.

21. Miele L, Osborne B. 1999. Arbiter of differentiation and death: Notch signaling meets apoptosis. J Cell Physiol 181:393-409.

22. Chillakuri CR, Sheppard D, Lea SM, Handford PA. 2012. Notch receptor-ligand binding and activation: insights from molecular studies. Semin Cell Dev Biol 23:421-8.

23. D'Souza B, Miyamoto A, Weinmaster G. 2008. The many facets of Notch ligands. Oncogene 27:5148-67.

24. Edwards RJ, Davey NE, O'Brien K, Shields DC. 2012. Interactome-wide prediction of short, disordered protein interaction motifs in humans. Mol Biosyst 8:282-95.

25. Kumar M, Gouw M, Michael S, Samano-Sanchez H, Pancsa R, Glavina J, Diakogianni A, Valverde JA, Bukirova D, Calyseva J, Palopoli N, Davey NE, Chemes LB, Gibson TJ. 2020. ELM-the eukaryotic linear motif resource in 2020. Nucleic Acids Res 48:D296D306.

26. Van Roey K, Uyar B, Weatheritt RJ, Dinkel H, Seiler M, Budd A, Gibson TJ, Davey NE. 2014. Short linear motifs: ubiquitous and functionally diverse protein interaction modules directing cell regulation. Chem Rev 114:6733-78.

27. Davey NE, Van Roey K, Weatheritt RJ, Toedt G, Uyar B, Altenberg B, Budd A, Diella F, Dinkel H, Gibson TJ. 2012. Attributes of short linear motifs. Mol Biosyst 8:268-81.

28. Dunphy PS, Luo T, McBride JW. 2014. Ehrlichia chaffeensis exploits host SUMOylation pathways to mediate effector-host interactions and promote intracellular survival. Infect Immun 82:4154-68.

29. Davey NE, Trave G, Gibson TJ. 2011. How viruses hijack cell regulation. Trends Biochem Sci 36:159-69.

30. Via A, Uyar B, Brun C, Zanzoni A. 2015. How pathogens use linear motifs to perturb host cell networks. Trends Biochem Sci 40:36-48.

31. Singh SB, Coffman CN, Carroll-Portillo A, Varga MG, Lin HC. 2021. Notch Signaling Pathway Is Activated by Sulfate Reducing Bacteria. Front Cell Infect Microbiol 11:695299.

32. Drayman N, Glick Y, Ben-nun-shaul O, Zer H, Zlotnick A, Gerber D, Schueler-Furman O, Oppenheim A. 2013. Pathogens use structural mimicry of native host ligands as a mechanism for host receptor engagement. Cell Host Microbe 14:63-73.

33. Mondino S, Schmidt S, Buchrieser C. 2020. Molecular Mimicry: a Paradigm of HostMicrobe Coevolution Illustrated by Legionella. mBio 11.

34. Price CT, Al-Khodor S, Al-Quadan T, Santic M, Habyarimana F, Kalia A, Kwaik YA. 2009. Molecular mimicry by an F-box effector of Legionella pneumophila hijacks a conserved polyubiquitination machinery within macrophages and protozoa. PLoS Pathog 5:e1000704.

35. Chemes LB, de Prat-Gay G, Sanchez IE. 2015. Convergent evolution and mimicry of protein linear motifs in host-pathogen interactions. Curr Opin Struct Biol 32:91-101.

36. Dunphy PS, Luo T, McBride JW. 2013. Ehrlichia moonlighting effectors and interkingdom interactions with the mononuclear phagocyte. Microbes Infect 15:1005-16. 
678

679

680

681

682

683

684

685

686

687

688

37. Keewan E, Naser SA. 2020. Notch-1 Signaling Modulates Macrophage Polarization and Immune Defense against Mycobacterium avium paratuberculosis Infection in Inflammatory Diseases. Microorganisms 8.

38. Veljkovic V, Vergara-Alert J, Segales J, Paessler S. 2020. Use of the informational spectrum methodology for rapid biological analysis of the novel coronavirus 2019-nCoV: prediction of potential receptor, natural reservoir, tropism and therapeutic/vaccine target. F1000Res 9:52.

39. Luca VC, Kim BC, Ge C, Kakuda S, Wu D, Roein-Peikar M, Haltiwanger RS, Zhu C, Ha T, Garcia KC. 2017. Notch-Jagged complex structure implicates a catch bond in tuning ligand sensitivity. Science 355:1320-1324.

40. Whiteman P, de Madrid BH, Taylor P, Li D, Heslop R, Viticheep N, Tan JZ, Shimizu H, Callaghan J, Masiero M, Li JL, Banham AH, Harris AL, Lea SM, Redfield C, Baron M, Handford PA. 2013. Molecular basis for Jagged-1/Serrate ligand recognition by the Notch receptor. J Biol Chem 288:7305-12.

41. Bruckner A, Polge C, Lentze N, Auerbach D, Schlattner U. 2009. Yeast two-hybrid, a powerful tool for systems biology. Int J Mol Sci 10:2763-88.

42. Andrawes MB, Xu X, Liu H, Ficarro SB, Marto JA, Aster JC, Blacklow SC. 2013. Intrinsic selectivity of Notch 1 for Delta-like 4 over Delta-like 1. J Biol Chem 288:25477-25489.

43. Kershaw NJ, Church NL, Griffin MD, Luo CS, Adams TE, Burgess AW. 2015. Notch ligand delta-like1: X-ray crystal structure and binding affinity. Biochem J 468:159-66.

44. Cordle J, Johnson S, Tay JZ, Roversi P, Wilkin MB, de Madrid BH, Shimizu H, Jensen S, Whiteman P, Jin B, Redfield C, Baron M, Lea SM, Handford PA. 2008. A conserved face of the Jagged/Serrate DSL domain is involved in Notch trans-activation and cisinhibition. Nat Struct Mol Biol 15:849-57.

45. Wang MM. 2011. Notch signaling and Notch signaling modifiers. Int J Biochem Cell Biol 43:1550-62.

46. Meng H, Zhang X, Hankenson KD, Wang MM. 2009. Thrombospondin 2 potentiates notch3/jagged1 signaling. J Biol Chem 284:7866-74.

47. Hu QD, Ang BT, Karsak M, Hu WP, Cui XY, Duka T, Takeda Y, Chia W, Sankar N, Ng YK, Ling EA, Maciag T, Small D, Trifonova R, Kopan R, Okano H, Nakafuku M, Chiba S, Hirai H, Aster JC, Schachner M, Pallen CJ, Watanabe K, Xiao ZC. 2003. F3/contactin acts as a functional ligand for Notch during oligodendrocyte maturation. Cell 115:163-75.

48. Ijdo JW, Carlson AC, Kennedy EL. 2007. Anaplasma phagocytophilum AnkA is tyrosinephosphorylated at EPIYA motifs and recruits SHP-1 during early infection. Cell Microbiol 9:1284-1296.

49. Habyarimana F, Price CT, Santic M, Al-Khodor S, Kwaik YA. 2010. Molecular characterization of the Dot/lcm-translocated AnkH and AnkJ eukaryotic-like effectors of Legionella pneumophila. Infect Immun 78:1123-34.

50. Huang L, Boyd D, Amyot WM, Hempstead AD, Luo ZQ, O'Connor TJ, Chen C, Machner $M$, Montminy T, Isberg RR. 2011. The E Block motif is associated with Legionella pneumophila translocated substrates. Cell Microbiol 13:227-45.

51. Gomez-Valero L, Rusniok C, Cazalet C, Buchrieser C. 2011. Comparative and functional genomics of legionella identified eukaryotic like proteins as key players in host-pathogen interactions. Front Microbiol 2:208.

52. Ravi Chandra B, Gowthaman R, Akhouri RR, Gupta D, Sharma A. 2004. Distribution of proline-rich (PxxP) motifs in distinct proteomes: functional and therapeutic implications for malaria and tuberculosis. Protein Eng Des Sel 17:175-82.

53. Klein T, Brennan K, Arias AM. 1997. An intrinsic dominant negative activity of serrate that is modulated during wing development in Drosophila. Dev Biol 189:123-34. 
54. Semenova D, Bogdanova M, Kostina A, Golovkin A, Kostareva A, Malashicheva A. 2020. Dose-dependent mechanism of Notch action in promoting osteogenic differentiation of mesenchymal stem cells. Cell Tissue Res 379:169-179.

55. Gomez-Lamarca MJ, Falo-Sanjuan J, Stojnic R, Abdul Rehman S, Muresan L, Jones ML, Pillidge Z, Cerda-Moya G, Yuan Z, Baloul S, Valenti P, Bystricky K, Payre F, O'Holleran K, Kovall R, Bray SJ. 2018. Activation of the Notch Signaling Pathway In Vivo Elicits Changes in CSL Nuclear Dynamics. Dev Cell 44:611-623 e7.

56. Cornell M, Evans DA, Mann R, Fostier M, Flasza M, Monthatong M, Artavanis-Tsakonas S, Baron M. 1999. The Drosophila melanogaster Suppressor of deltex gene, a regulator of the Notch receptor signaling pathway, is an E3 class ubiquitin ligase. Genetics 152:567-76.

57. Hambleton S, Valeyev NV, Muranyi A, Knott V, Werner JM, McMichael AJ, Handford PA, Downing AK. 2004. Structural and functional properties of the human notch-1 ligand binding region. Structure 12:2173-83.

58. Maveyraud L, Mourey L. 2020. Protein X-ray Crystallography and Drug Discovery. Molecules 25.

59. Luo T, Zhang X, McBride JW. 2009. Major species-specific antibody epitopes of the Ehrlichia chaffeensis p120 and E. canis p140 orthologs in surface-exposed tandem repeat regions. Clin Vaccine Immunol 16:982-90.

60. Deng SP, Huang DS. 2014. SFAPS: an R package for structure/function analysis of protein sequences based on informational spectrum method. Methods 69:207-12.

61. Doyle CK, Nethery KA, Popov VL, McBride JW. 2006. Differentially expressed and secreted major immunoreactive protein orthologs of Ehrlichia canis and E. chaffeensis elicit early antibody responses to epitopes on glycosylated tandem repeats. Infect Immun 74:711-20.

62. Luo T, Zhang X, Wakeel A, Popov VL, McBride JW. 2008. A variable-length PCR target protein of Ehrlichia chaffeensis contains major species-specific antibody epitopes in acidic serine-rich tandem repeats. Infect Immun 76:1572-80. 
Fig. 1. E. chaffeensis TRP120 shares sequence homology and biological function with

canonical and noncanonical Notch ligands. (A) BLAST analysis of TRP120 with represents identical conserved amino acid residues; a colon (:) represents conservative substitutions. (B) Informational Spectrum Method (ISM) was used to predict if TRP120 shared similar biological function with canonical and nonocanonical Notch ligands. Primary sequences of TRP120 and Notch ligands were converted into a numerical sequence-based electron ion interaction potential (EIIP) of each amino acid. Numerical sequences were converted into a spectrum using Fourier transform. To determine if proteins shared a similar biological function four highlighted repetitive TRP120 TR motifs that share sequence homology with Notch ligands. ISM sequence shown in panel B (underlined). $\left(^{*}\right)$ represents a partial tandem repeat containing similar (EDDTVSQPSLE) but non identical sequence to highlighted sequence.

Fig. 2. E. chaffeensis TRP120-TR interactions with the Notch receptor ligand binding region (LBR). (A) HeLa cells transfected with TRP120-GFP (green) and probed for endogenous Notch-1 (red) demonstrate colocalization by immunofluorescent microscopy.

736 Colocalization was quantitated by Pearson's and Mander's coefficient ( -1 no colocalization; +1

737 strong colocalization). (B and $\mathrm{C}$ ) His-tag pull down assays demonstrating direct interaction 738 between TRP120 and Notch-1. Recombinant Fc-tagged Notch-1 LBR was incubated with (B) 739 TRP120-FL-His, (C) TRP120-TR-His or TRX-His negative control on Talon metal affinity resin. 740 Bound Notch-1, TRP120-His, $\alpha$-TRP120 against a TR peptide or TRX-His were detected with $\alpha$ 741 Notch-1, $\alpha$-TRP120 or $\alpha-T R X$ antibodies. (D-F) Surface plasmon resonance of (D) TRP120-FL742 His, (E) TRP120-TR-His or (F) TRX-His with Fc-tagged Notch-1 LBR on a Biacore T100 with a 
series S Ni-nitrilotriacetic acid (NTA) sensor chip. TRP120-FL-His, TRP120-TR-His or TRX-His were immobilized on the NTA chip and 2-fold dilutions (800nM to $25 \mathrm{nM})$ of Fc-tagged Notch-1 LBR were used as analyte to determine binding affinity $\left(K_{D}\right)$. Sensograms and $K_{D}$ are representative of data from triplicate experiments. (G) THP-1 cells were treated with rTRX- or rTRP120-FL-coated fluorescent microspheres for varying time points (5-60 mins). Colocalization was visualized by confocal immunofluorescent microscopy. Notch-1 was immunostained with tetramethylrhodamine isothiocyanate [TRITC] and TRP120-coated fluorescein isothiocyanate [FITC] auto-fluorescent microspheres. Nuclei were stained with DAPI (blue). White boxes indicate areas of colocalization measurements. Scale bar $=10 \mu \mathrm{m} .(\mathrm{H})$ Dot blot of PBS, TRX or TRP120-FL-coated microspheres probed with $\alpha$-TRX or $\alpha$-TRP120 antibodies, respectively.

\section{Fig. 3. TRP120-TR activates Notch and NICD nuclear translocation in primary human} monocytes. (A) Soluble recombinant TRP120-TR or -C terminal proteins $(2 \mu \mathrm{g} / \mathrm{ml})$ were incubated with THP-1 cells for 2 h. Cells were collected and NICD localization determined by confocal immunofluorescent microscopy. Uninfected/untreated or recombinant TRX-treated THP-1 cells were used as negative controls. E. chaffeensis-infected or recombinant Jagged-1 treated THP-1 cells were used as positive controls. NICD nuclear translocation was detected in E. ch-infected, TRP120-TR and Jagged-1 treated cells. (B) Primary human monocytes were treated with soluble TRP120-TR or recombinant TRX as described above and NICD nuclear translocation was detected in E. chaffeensis-infected and TRP120-TR-treated cells. End point analysis was performed as described in Fig. 2. Experiments were performed in triplicate and representative images are shown.

\section{Fig. 4. A TRP120-TR Notch-1 memetic IDD peptide stimulates NICD nuclear translocation.}

(A) Overlapping TRP120-TR IDD peptide sequences (P1-P3) (B) THP-1 cells or (C) Primary human monocytes were incubated with synthetic TRP120-TR IDD peptides to determine the 
TRP120-TR Notch-1 memetic motif responsible for Notch activation. TRP120-TR peptides were overlapping peptides spanning an entire TR domain. Cells were treated with peptide $(1 \mu \mathrm{g} / \mathrm{ml})$

771 for $2 \mathrm{~h}$ and confocal immunofluorescent microscopy was used to visualize NICD localization.

772 NICD nuclear translocation denotes Notch activation. A scrambled peptide (Ctrl-p) was used as 773 negative control and E.ch. infected cells were used as positive control. To determine if direct 774 interaction of the TRP120-N1-P3 peptide and Notch receptor was necessary for Notch 775 activation, THP-1 cells were pre-treated with DAPT, a y-secretase inhibitor, and treated with 776 TRP120-N1-P3 peptide for $2 \mathrm{~h}$.

Fig. 5. TRP120-N1-P3 IDD peptide stimulates Notch gene expression. (A) Table of Notch pathway genes with corresponding fold-change displaying differential expression (up, down or no change) at $24 \mathrm{~h}$ p.t with $10 \mathrm{ng} / \mathrm{ml}$ of TRP120-N1-P3 peptide (B) Scatter plots of expression array analysis of 84 Notch signaling pathway genes to determine Notch gene expression $24 \mathrm{~h}$ after stimulation with $1 \mathrm{ng} / \mathrm{ml}$ (top), $10 \mathrm{ng} / \mathrm{ml}$ (middle) or $100 \mathrm{ng} / \mathrm{ml}$ (bottom) of TRP120-N1-P3 peptide. Purple lines denote a 2 -fold up or down regulation in comparison to control, and the black line denotes no change. Scatter plots are representative of three independent experiments $(n=3)$. TRP120-N1 SLiM (P4-P6) and mutant (dmut) peptide sequences. (B) THP-1 cells or (C) primary human monocytes were treated with synthetic TRP120-TR SLiM peptides to identify the 790 TRP120-TR Notch-1 SLiM memetic motif. TRP120-TR peptides were SLiM peptides spanning 791 the entire TRP120-N1-P3 peptide sequence. TRP120-N1-P3 mutant peptide (dmut) has a 792 deletion of the TRP120-N1-P6 amino acids. Cells were treated with peptide $(1 \mu \mathrm{g} / \mathrm{ml})$ for $2 \mathrm{~h}$ and 793 NICD localization visualized by confocal microscopy. TRP120-N1-P3 peptide was used as a 794 positive control. To determine if direct interaction of the TRP120-N1-P6 peptide and Notch 
795

796

797

798

799

800

801

802

803

804

805

806

807

808

809

810

811

812

813

814

815

816

817

818

819

820

receptor was necessary for Notch activation, THP-1 cells were pre-treated with DAPT, a $\mathrm{Y}^{-}$ secretase inhibitor, and treated with TRP120-N1-P6 peptide for $2 \mathrm{~h}$. Representative data of all experiments are shown $(n=3)$.

\section{Fig. 7. TRP120-N1-P6 SLiM Notch memetic peptide stimulates Notch gene expression. (A)}

Selected Notch pathway genes with corresponding fold-change displaying differential expression (up- and downregulation) at $24 \mathrm{~h}$ p.t with $10 \mathrm{ng} / \mathrm{ml}$ of TRP120-N1-P6 peptide. (B) Scatter plots of expression array analysis of 84 Notch signaling pathway genes to determine Notch gene expression with $1 \mathrm{ng} / \mathrm{ml}, 10 \mathrm{ng} / \mathrm{ml}, 100 \mathrm{ng} / \mathrm{ml}$ of TRP120-N1-P6 peptide or TRP120N1-P5 treatment (10 ng/ml) compared to untreated cells (bottom) at 24 p.t. Purple lines denote a 2-fold up or down regulation in comparison to control, and the black lines denotes no change. Scatter plots are representative of three independent experiments $(n=3)$.

Fig. 8. Amino acids critical to TRP120-N1-P6 memetic SLiM activity and anti-SLiM antibody blocks Notch activation (A) Critical amino acids of the TRP120-N1-P6 memetic SLiM determined by alanine mutagenesis (mutant peptide sequences are shown above the corresponding panel). THP-1 cells were treated with mutant peptides (dmut2, -3 and $-4 ; 1 \mu \mathrm{g} / \mathrm{ml}$ ) for $2 \mathrm{~h}$ and confocal immunofluorescent microscopy was used to visualize NICD localization. NICD nuclear translocation denotes Notch activation. Peptide dmut was used as a negative control and TRP120-N1-P6 was used as a positive control. (B) Cell-free E. chaffeensis, rTRP120-FL or TRP120-N1-P6 were incubated with $\alpha$-TRP120-N1-P6 rabbit polyclonal antibody $(5 \mu \mathrm{g} / \mathrm{ml})$ for $30 \mathrm{~min}$. Preimmune serum was used as control antibody. THP-1 cells were subsequently inoculated with the cell-free E. chaffeensis/a-TRP120-N1-P6 mixture for $2 \mathrm{~h}$ and confocal immunofluorescent microscopy was used to visualize NICD nuclear localization. Representative data of all experiments are shown $(n=3)$. 

SLiM memetic motif (TRP120-N1-P6; yellow highlight) binds the Notch-1 extracellular domain at a region containing the confirmed Notch ligand binding domain (LBD) to activate Notch signaling. TRP120-N1-P6 binding leads to NICD nuclear translocation and upregulation of

$825 \quad$ Notch gene targets.

Fig. S1. TRP120 shares biological function with canonical and noncanonical Notch ligands. Informational Spectrum Method (ISM) was used to predict if TRP120 shared similar biological function with endogenous canonical and noncanonical Notch ligands. The primary sequence of TRP120 and endogenous Notch ligands were converted into a numerical sequence using each amino acids electron ion interaction potential (EIIP). Numerical sequences were converted into a spectrum using Fourier transform. To determine if proteins shared a similar biological function and cross spectra analysis was performed with TRP120 and Notch ligands DLL3 and (C) DLL4 and (D) noncanonical Notch ligand, F3 Contactin-1.

Fig. S2. Purification of recombinant TRP120 proteins. (A) Schematic of TRP120-FL, -TR and -C-terminus recombinant proteins. TRP120-TR is expressed and purified as two tandem repeat domains. (B) Coomassie Blue stained gel displaying expression of purified TRP120-FL, -

$841 \mathrm{TR},-\mathrm{N},-\mathrm{C}$-terminus and TRX recombinant proteins. All listed recombinant proteins were 842 expressed in a pBAD vector containing a His-tag.

Fig. S3. Jagged-1 activates Notch gene expression in a concentration-dependent manner.

845 Scatter plots of expression array analysis of 84 Notch signaling pathway genes to determine 846 Notch gene expression with $1 \mathrm{ng} / \mathrm{ml}$ (top left). $10 \mathrm{ng} / \mathrm{ml}$ (top right) $100 \mathrm{ng} / \mathrm{ml}$ (bottom left) of 
bioRxiv preprint doi: https://doi.org/10.1101/2022.01.13.476283; this version posted January 15, 2022. The copyright holder for this preprint (which was not certified by peer review) is the author/funder. All rights reserved. No reuse allowed without permission.

847 recombinant Jagged-1 at 24 p.t. Purple lines denote a 2-fold up or down regulation in 848 comparison to control, and the black lines denotes no change. 
A.

\begin{tabular}{|c|c|}
\hline $\begin{array}{r}\text { TRP120 } \\
\text { Jagged-1 }\end{array}$ & 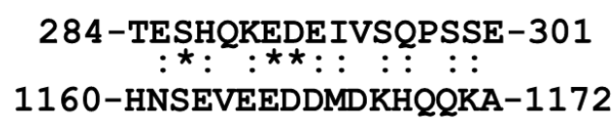 \\
\hline TRP120 & 284-TESHQKEDEIVSOPSSE-301 \\
\hline DLL1 & 655-RDA் SKRDTK-CQPQG் -671 \\
\hline TRP120 & 284-TESHQKEDEIVSQPSSE-301 \\
\hline DLL4 & 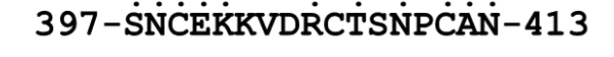 \\
\hline TRP120 & 284-TESHQKEDEIVSQPSSE-301 \\
\hline TSP2 & 970-VIRHQGKं-ELVQTANSD-999 \\
\hline
\end{tabular}

C.

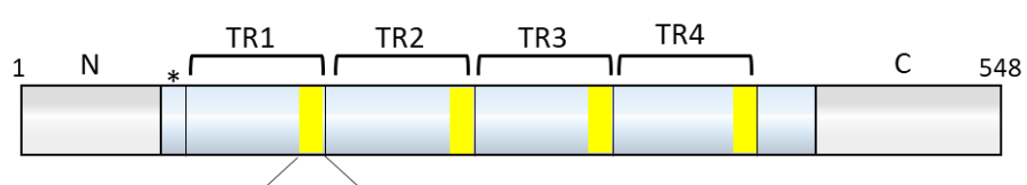

TESHQKEDEIVSQPSSE

$\square$ BLAST Sequence Homology/ $\underline{\mathrm{ISM}}$
B. 214 -IVSOPSSEPFVAESEVSKVEQEETNPEVLIKDLQD - 248

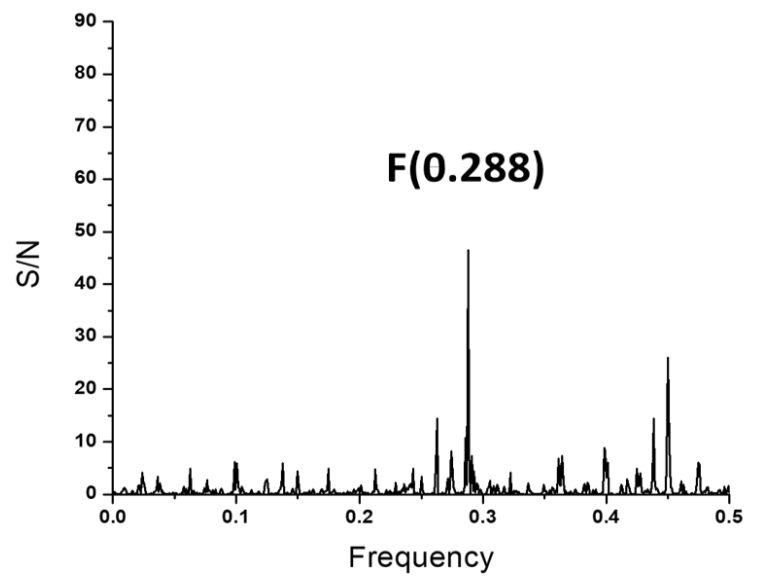


A.
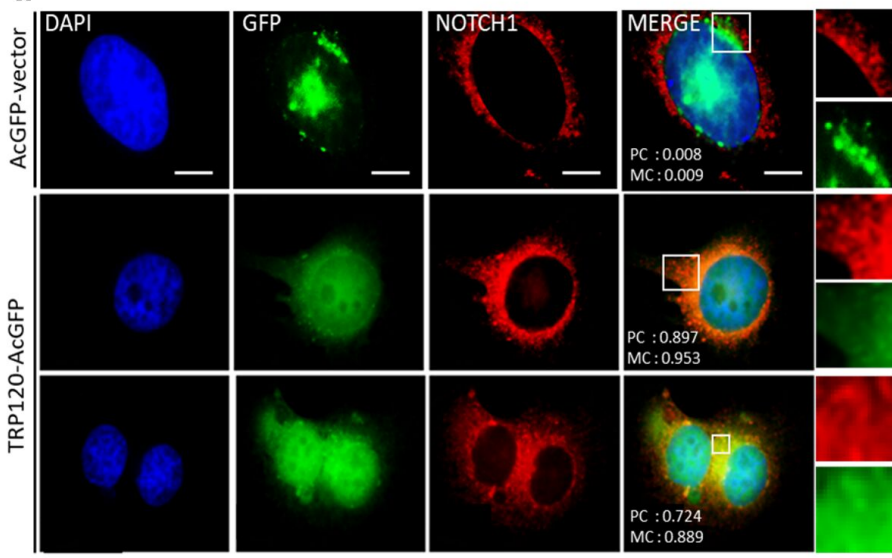

D.
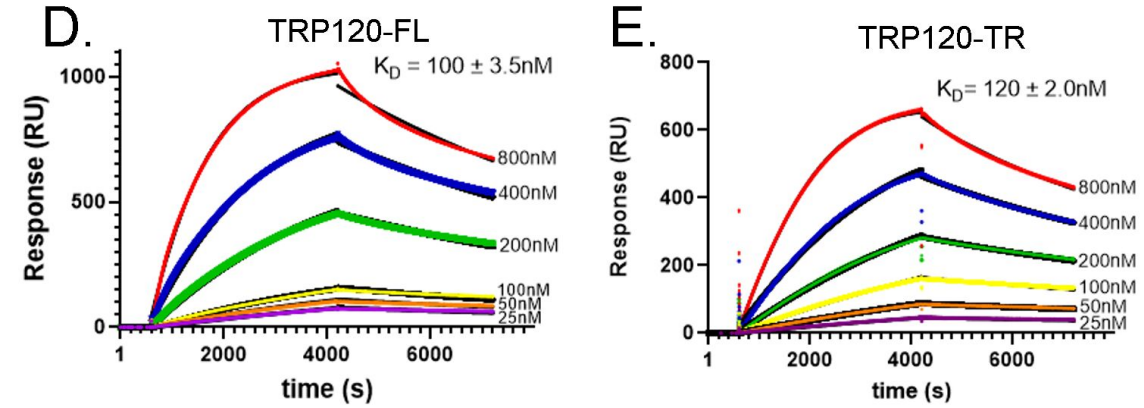

mins
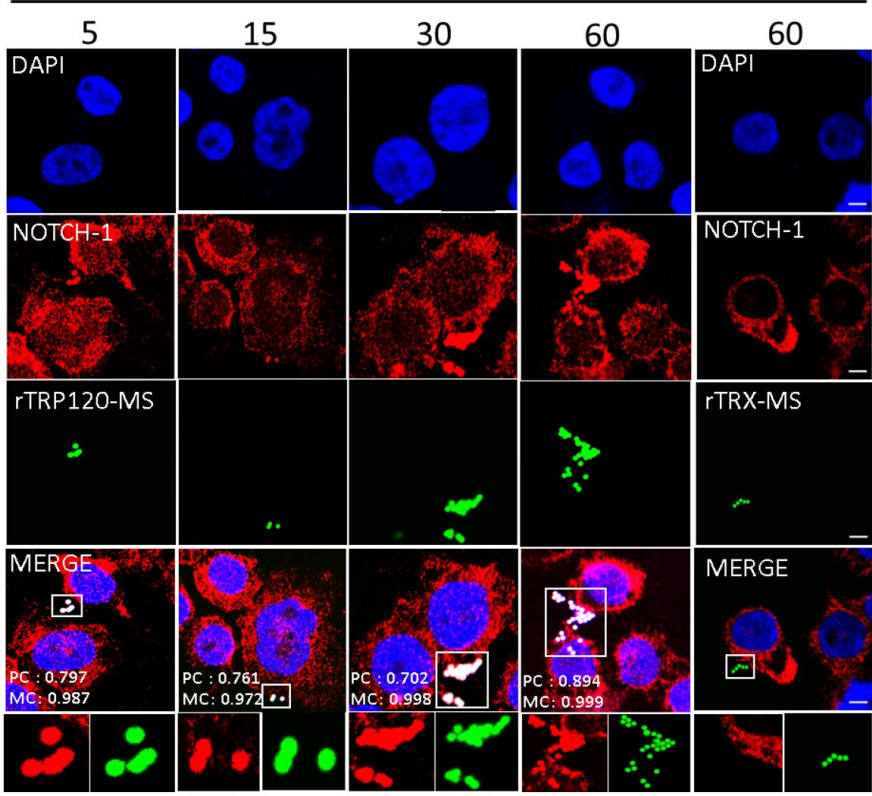

$\mathrm{H}$

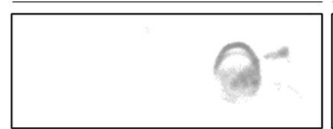

PBS-coated

TRX-coated fluorospheres fluorospheres

G.
B. ${ }_{\text {TRP120-FL }} \frac{\text { Input }}{+--+-\frac{\text { Pull-down }}{+--+}}$

Notch-1 -+-++-+++

TRX - - + + - + - +

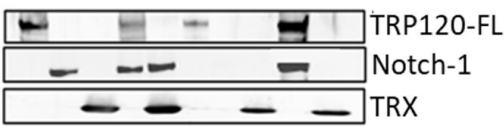

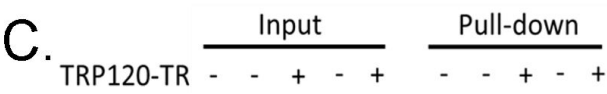

TRX - + - + - - + - + -

Notch-1 $+--_{-}+\quad+--_{+}+$

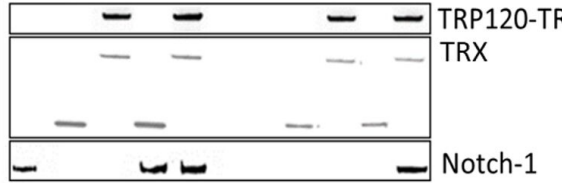

F.

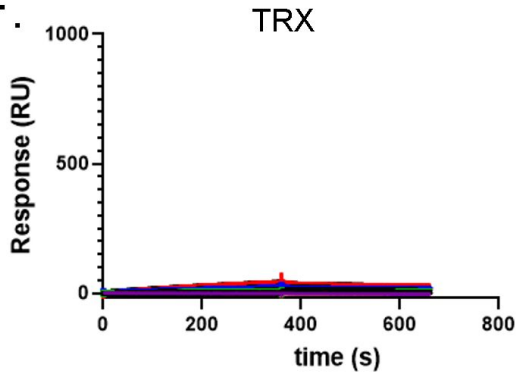


A.

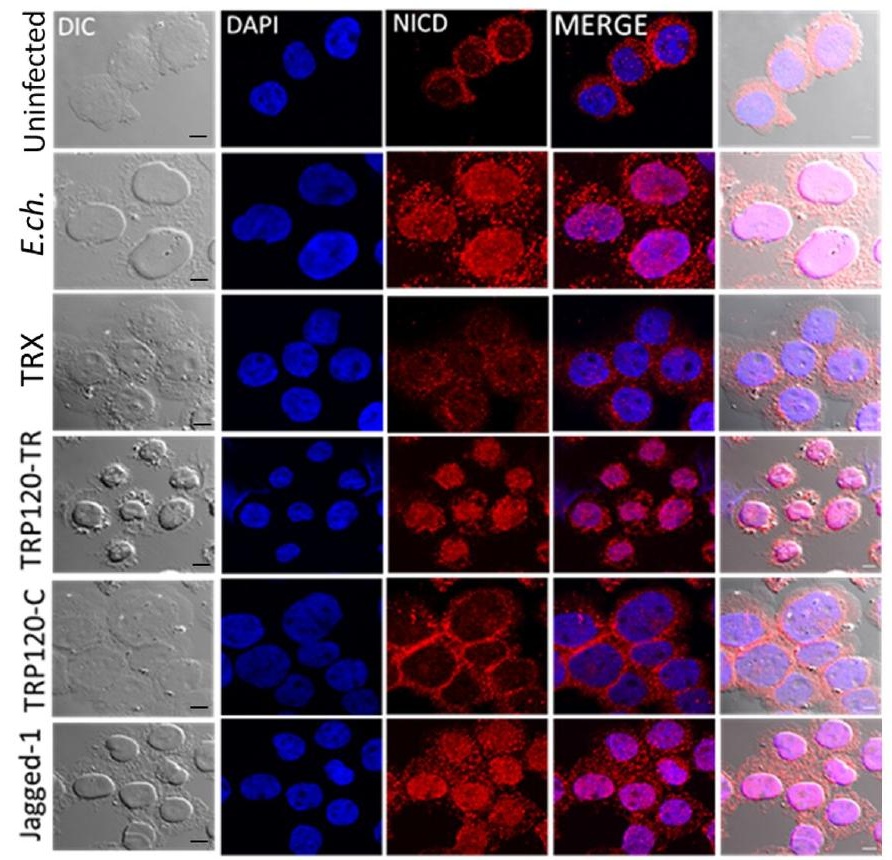

B.

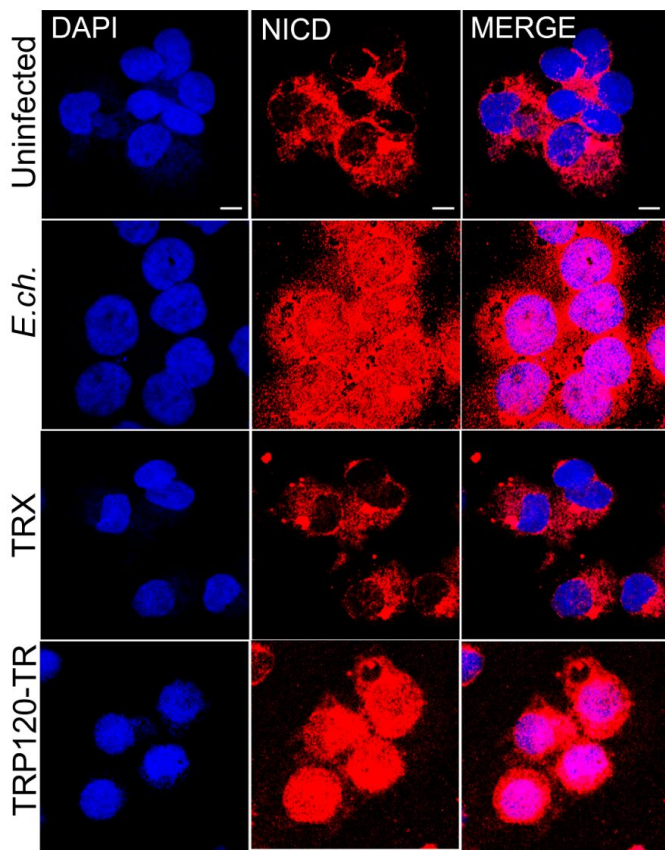


A. $\frac{\text { TRP120-N1-P1 }}{\text { PFVAESEVSKVEOEETNPEVII }}$

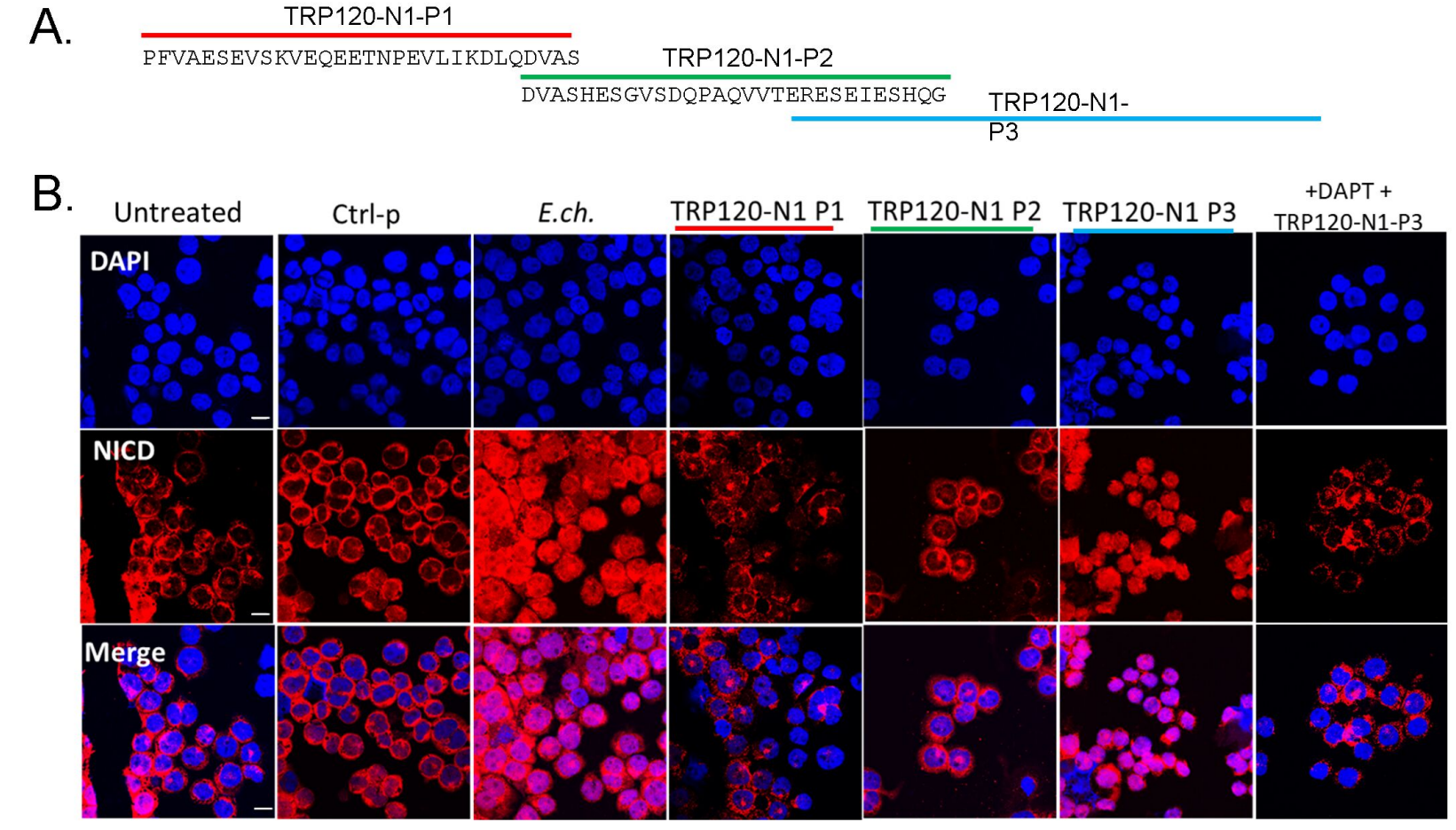

C

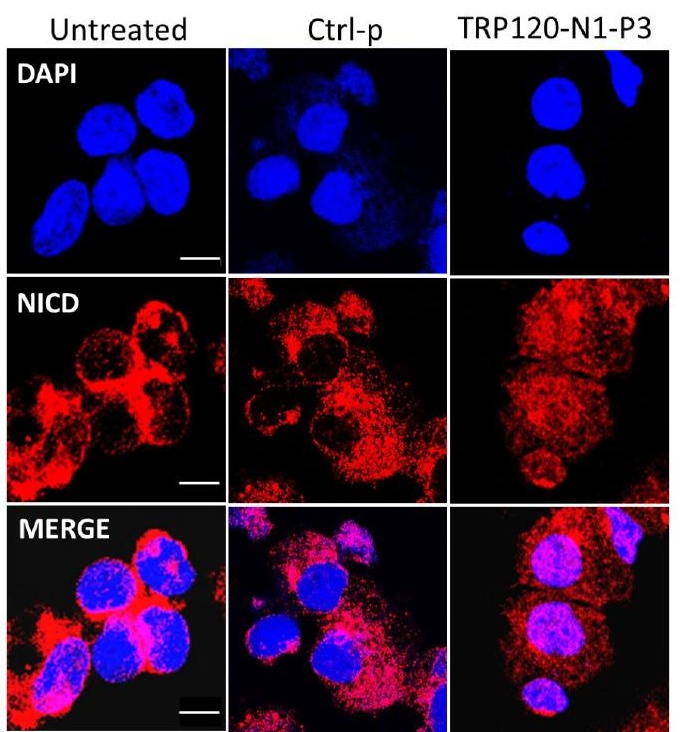




\section{A. Gene Symbol $\quad$ Fold Regulation}

\begin{tabular}{|c|c|}
\hline CD44 & 9.77 \\
\hline DLL1 & 11.16 \\
\hline DLL3 & 10.14 \\
\hline DLL4 & 436.97 \\
\hline DTX1 & 538.76 \\
\hline HES1 & 86.35 \\
\hline HES5 & 43.98 \\
\hline HEY1 & 108.88 \\
\hline HEY2 & 205.31 \\
\hline HEYL & 2.47 \\
\hline PAX5 & 1622.97 \\
\hline POFUT1 & 82.42 \\
\hline PPRAG & 46.65 \\
\hline PSEN1 & 64.59 \\
\hline PTCRA & -1.94 \\
\hline SH2D1A & -7.67 \\
\hline ADAM17 & 9.84 \\
\hline JAG1 & 654.18 \\
\hline JAG2 & 90.37 \\
\hline MAML1 & 32.92 \\
\hline MAML2 & 34.17 \\
\hline NOTCH2 & -2.43 \\
\hline NOTCH3 & 112.67 \\
\hline FZD4 & 18.40 \\
\hline GLI1 & 36.43 \\
\hline SUFU & 126.99 \\
\hline
\end{tabular}

Upregulated

Unchanged
B.
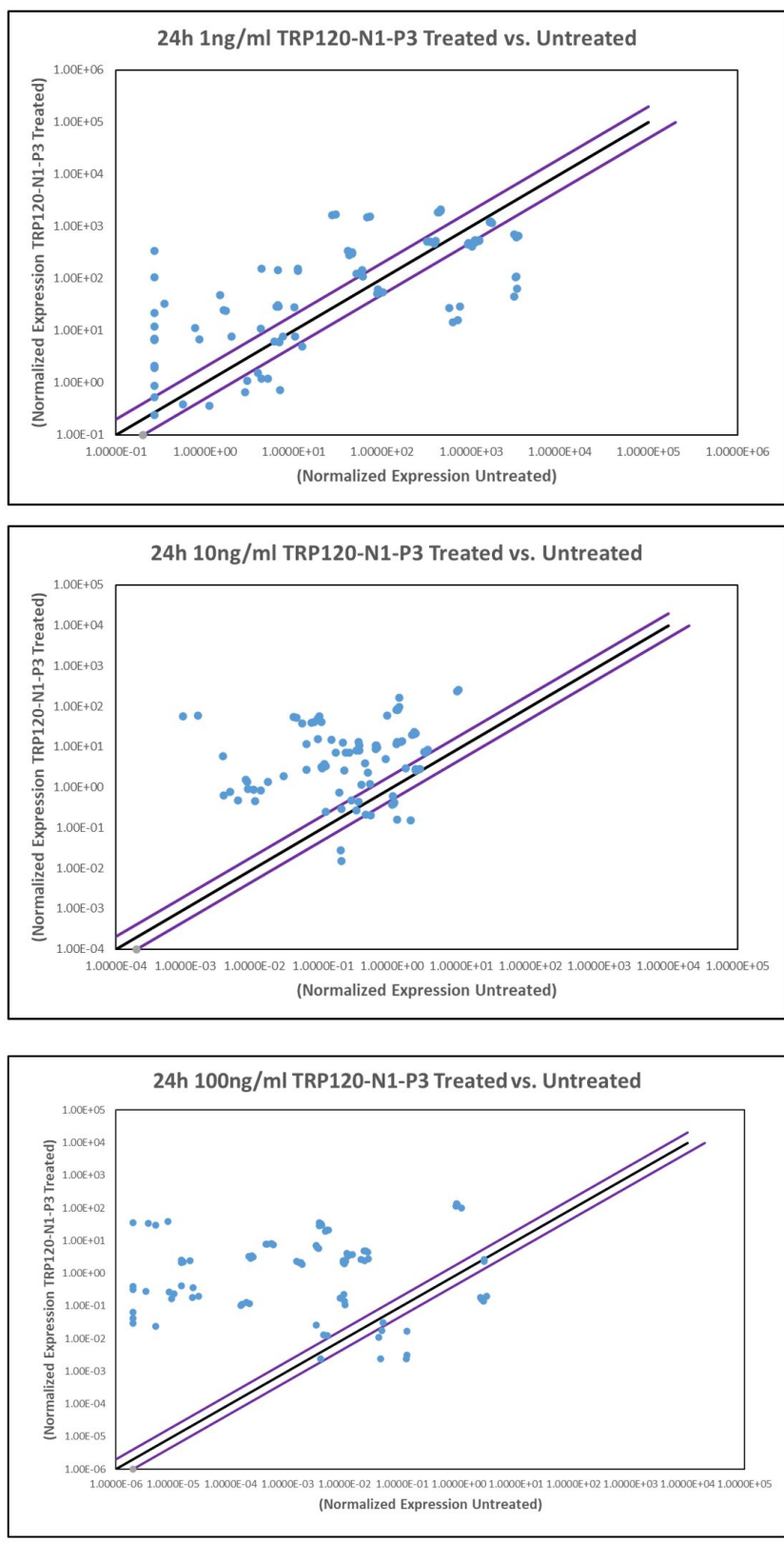
A.

TRP120-N1-P3

RESEIESHQGETEKESGITESHQKEDEIVSQPSSE

TRP120-N1-P4 TRP120-N1-P5 TRP120-N1-P6

TRP120-N1-dmut

+ DAPT +

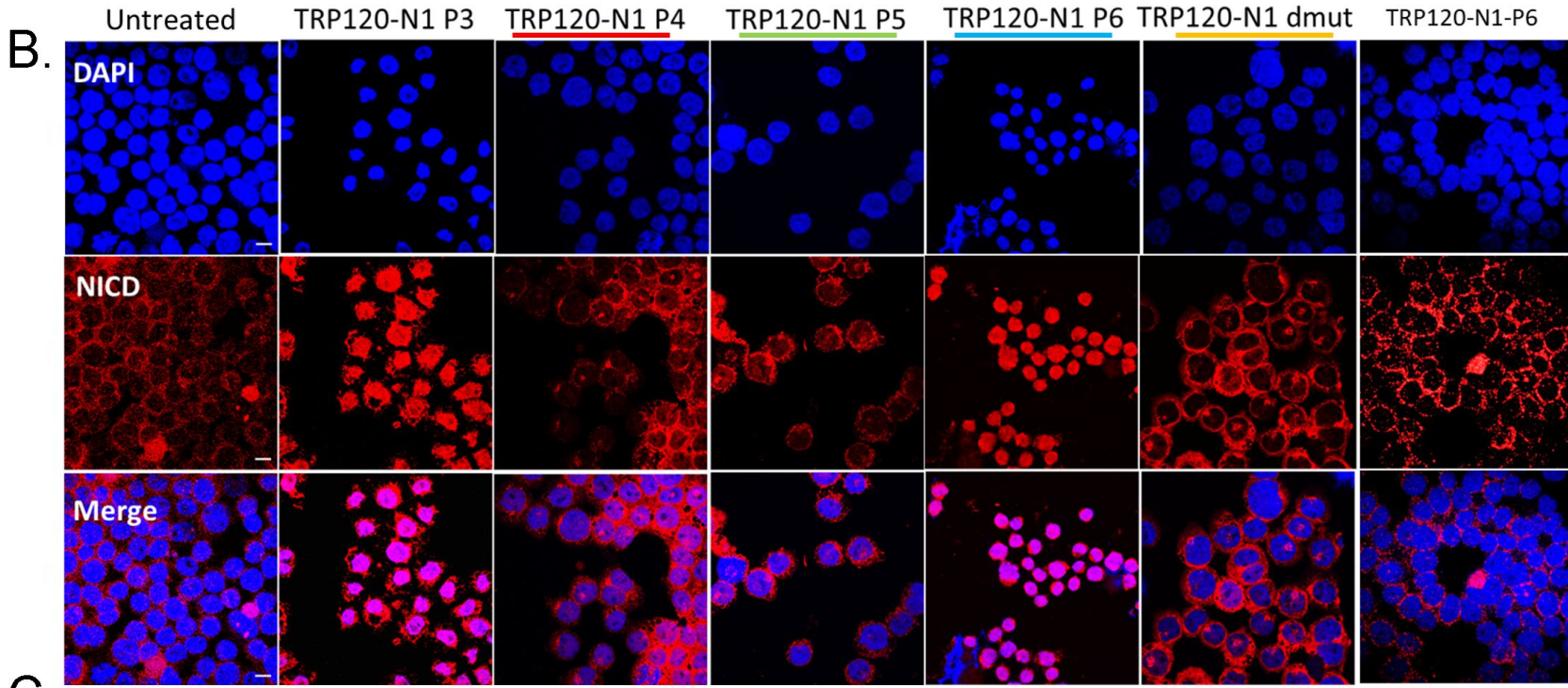

C

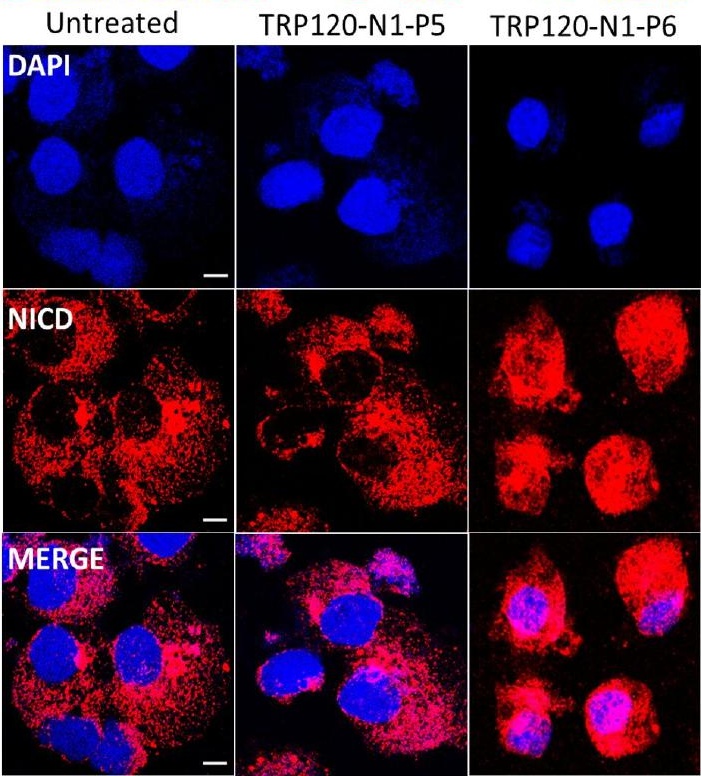


Fold Regulation

B.

\begin{tabular}{|l|l|}
\hline CD44 & 20.74 \\
\hline DLL1 & 15.53 \\
\hline DLL3 & 12.75 \\
\hline DLL4 & 407.22 \\
\hline DTX1 & 477.81 \\
\hline HES1 & 25.77 \\
\hline HES5 & 220.74 \\
\hline HEY1 & 145.40 \\
\hline HEY2 & 193.21 \\
\hline HEYL & 5.73 \\
\hline PAX5 & 349.38 \\
\hline POFUT1 & 177.47 \\
\hline
\end{tabular}

PPRAG

192.04

PSEN1

52.40

PTCRA

1.37

SH2D1A

$-8.36$

ADAM17

14.29

JAG1

JAG2

MAML1

46.73

723.27

MAML2

NOTCH2

NOTCH3

FZD4

22.17

GLI1

39.78

SUFU

157.74

\subsection{6}

223.35

22.17

157.74

Downregulated

Upregulated

Unchanged 24h 1ng/ml TRP120-N1-P6 Treated vs. Untreated

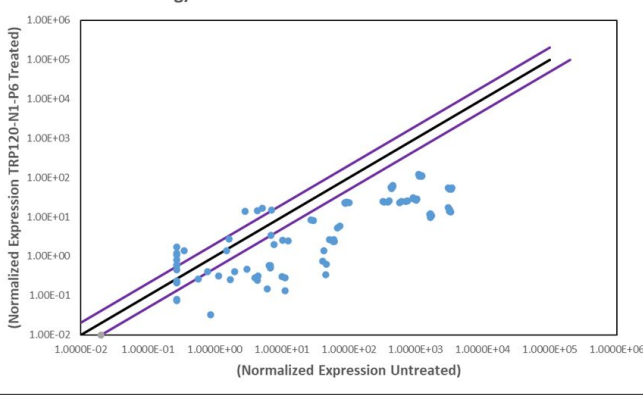

24h 10ng/ml TRP120-N1-P6 Treated vs. Untreated

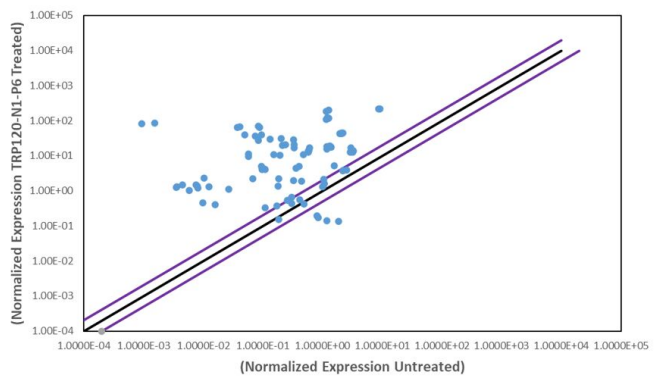

24h 100ng/ml TRP120-N1-P6 Treated vs. Untreated

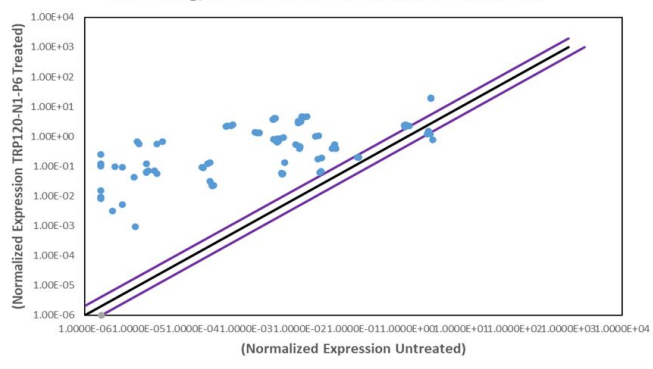

24h 10ng/ml TRP120-N1-P5 Treated vs. Untreated

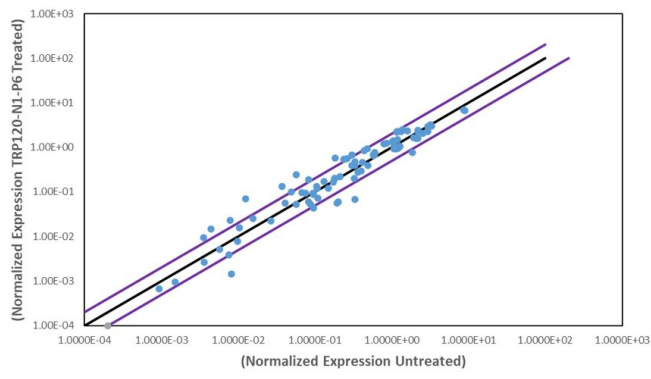


A.

TRP120-N1 TRP120-N1 TRP120-N1 TRP120-N1

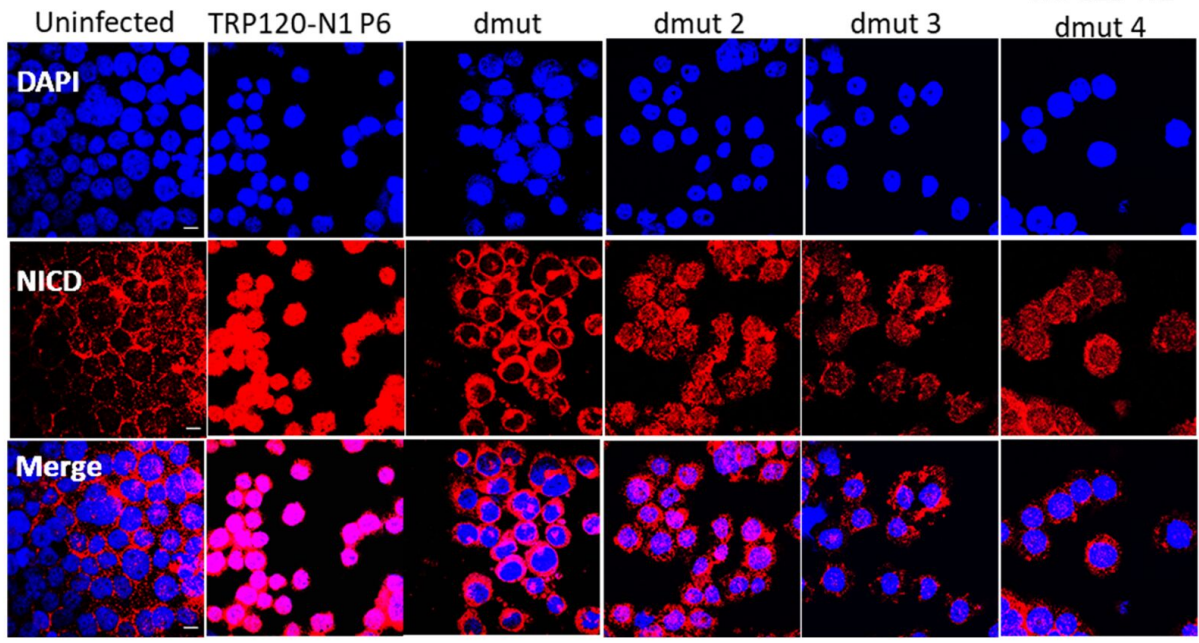

E.ch. +

B.
$\alpha$-TRP120-N1-P6

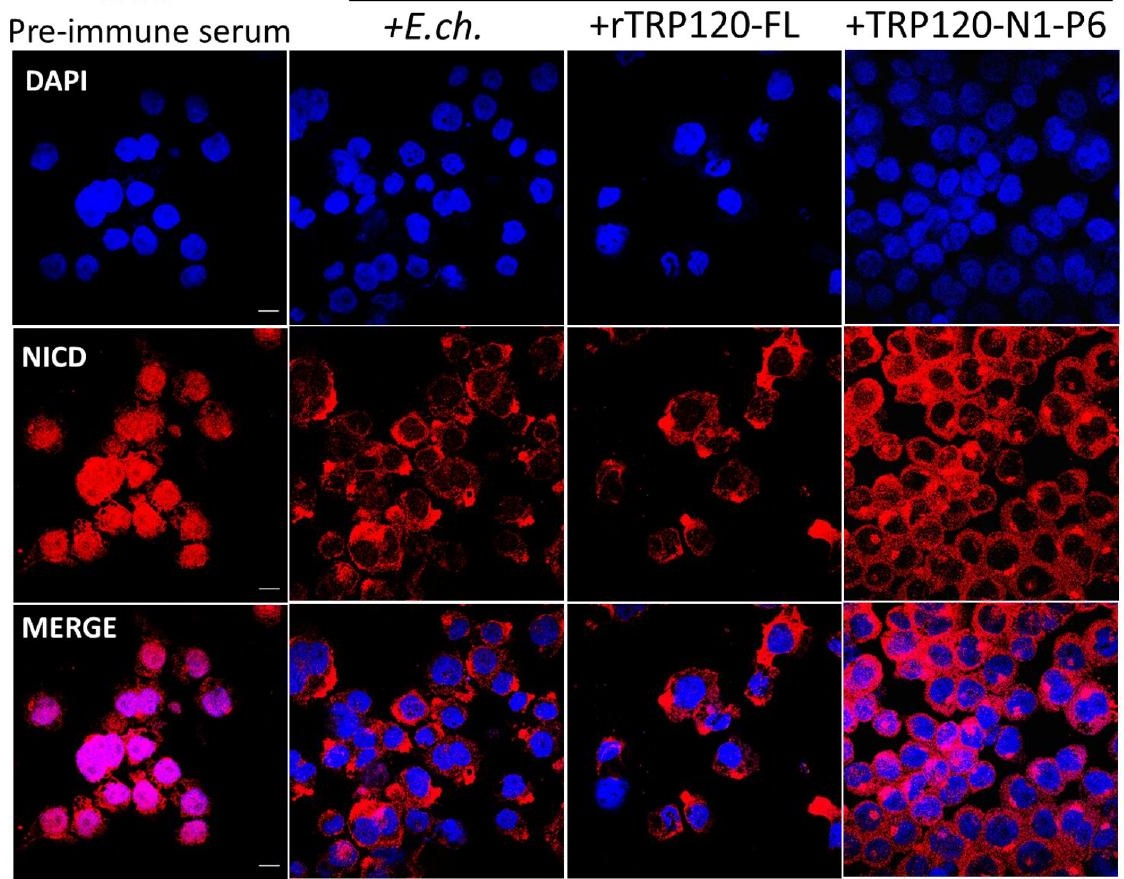


A.

TRP120 x DLL1
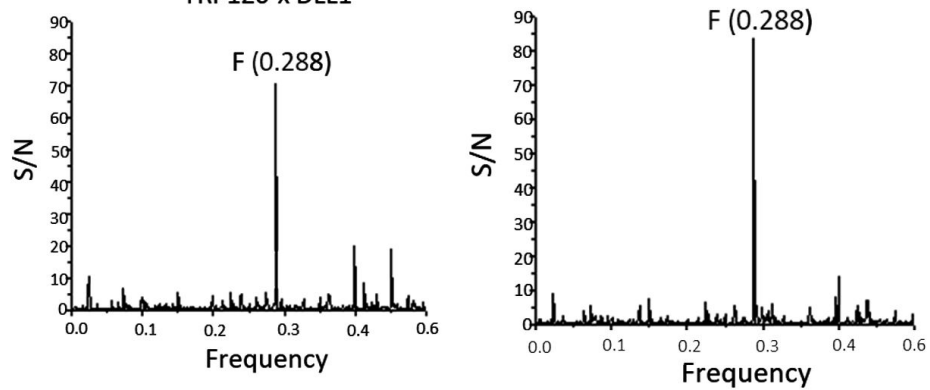

C.

TRP120 x DLL4
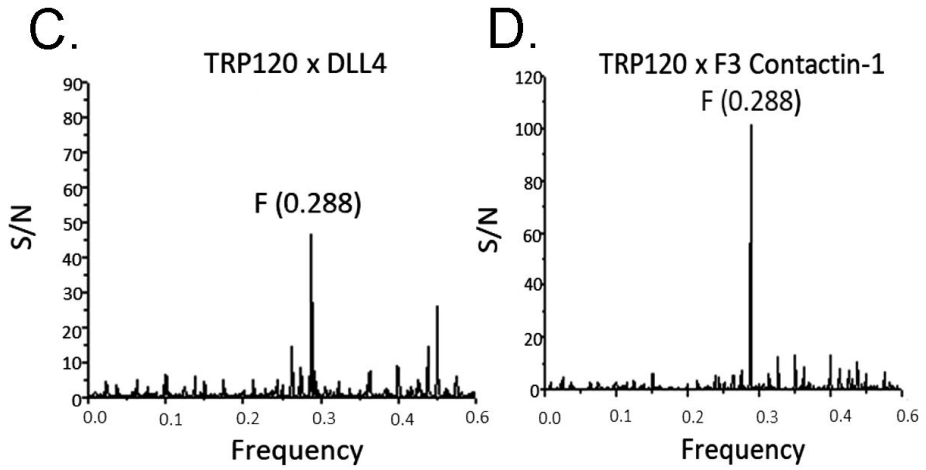

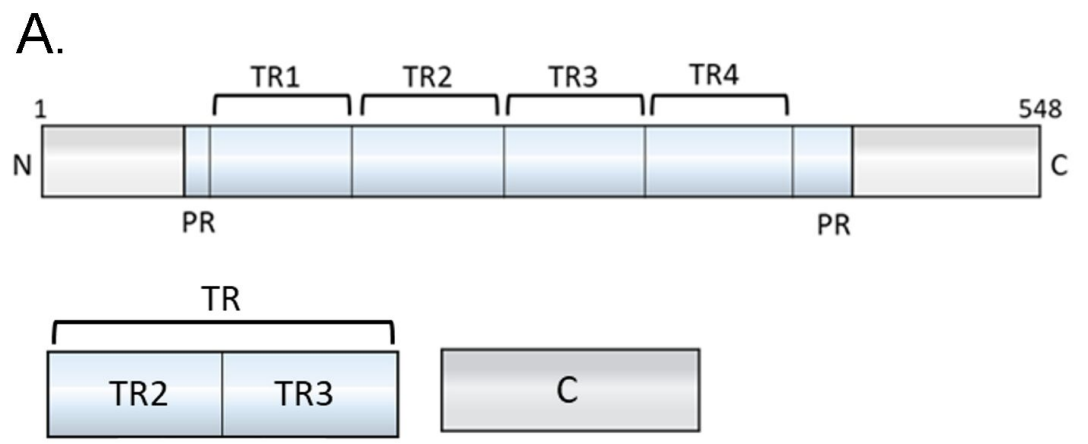

B.

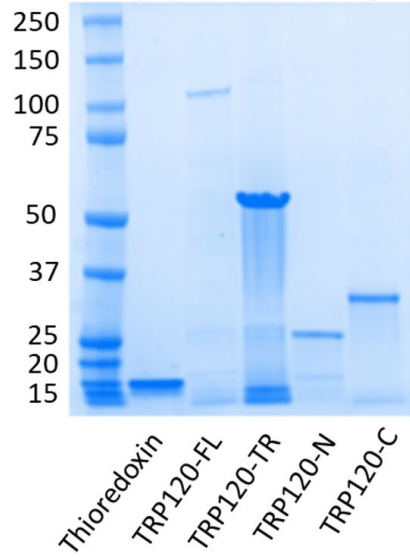

Expected Sizes:

Thioredoxin: $16 \mathrm{kDa}$

TRP120-FL: 120kDa

TRP120-TR: $52 \mathrm{kDa}$

TRP120-N: 23kDa

TRP120-C: 32kDa 
